\title{
Intersections of Random Walks. A Direct Renormalization Approach
}

\author{
Bertrand Duplantier \\ Service de Physique Théorique CEN Saclay, F-91191 Gif-sur-Yvette Cedex, France
}

\begin{abstract}
Various intersection probabilities of independent random walks in $d$ dimensions are calculated analytically by a direct renormalization method, adapted from polymer physics. This heuristic approach, based on Edwards' continuum model, leads to a straightforward derivation and also to refinements of Lawler's results for the simultaneous intersections of two walks in $\mathbb{Z}^{4}$, or three walks in $\mathbb{Z}^{3}$. These results are generalized to $P$ walks in $\mathbb{Z}^{d^{*}}, d^{*}=\frac{2 P}{P-1}, P \geqq 2$. For $d<4$, an infinite set of universal critical exponents $\sigma_{L}, L \geqq 1$, are derived. They govern the asymptotic probability $\mathscr{Z}_{L} \sim S^{\sigma_{L}}$ that $L$ "star walks" in $\mathbb{R}^{d}$, with a common origin, do not intersect before time $S$. The $\sigma_{L}$ 's are calculated up to order $O\left(\varepsilon^{2}\right)$, where $d=4-\varepsilon$. This information is used to calculate the probability $\mathscr{Z}(\mathscr{G})$ that a set of independent random walks in $\mathbb{R}^{d}$ or $\mathbb{Z}^{d}, d \leqq 4$, (respectively $d \leqq 3$ ) form a given topological networks $\mathscr{G}$ of multiple intersection points, in the absence of any other double point (respectively triple point). This is generalized to a network in $d \leqq \frac{2 P}{P-1}$ dimension with exclusion of $P$-tuple points. The method is quite general and can be used to calculate any critical intersection probability, and provides the probabilist with a large variety of exact results (yet to be proven rigorously).
\end{abstract}

\section{Table of Contents}

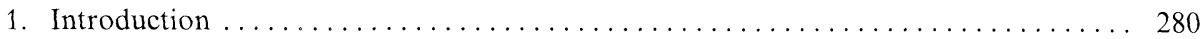

2. Intersections of Two Walks Near Four Dimensions . . . . . . . . . . . . . . . . . 284

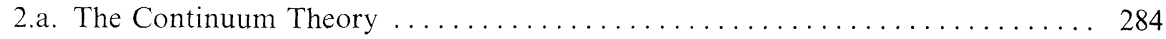

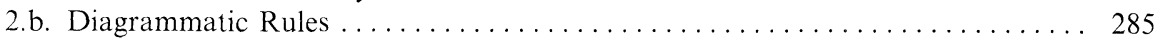

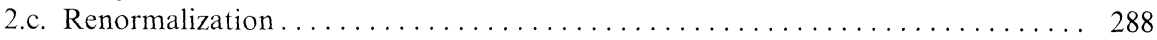

2.d. Other Geometrical Cases ... . . . . . . . . . . . . . . . . . . . . . . . . 291

3. Intersections of Three Walks Near Three Dimensions . . . . . . . . . . . . . . . . . . 297 3.a. Lawler's Results . . . . . . . . . . . . . . . . . . . . . . . . . . . . . . . 298 
3.b. The Three-Body Continuum Model and Its Renormalization ............ 299

3.c. Intersections of Three Walks . . . . . . . . . . . . . . . . . . . . . . . . . . . 301

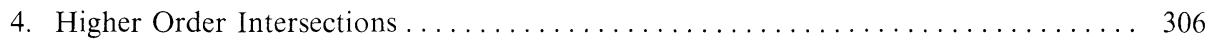

4.a. Model ................................................ 306

4.b. Renormalization .................................... 309

5. More Results Near Four Dimensions . . . . . . . . . . . . . . . . . . . . . . 311

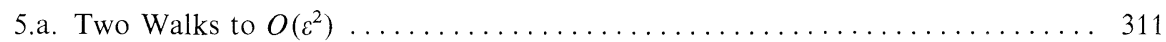

5.b. Renormalization ....................................... 313

5.c. Asymptotic Evaluation of the Probability of no Intersection . . . . . . . . . . 314

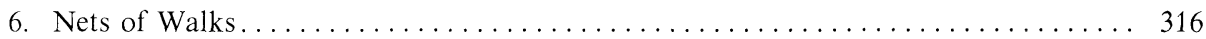

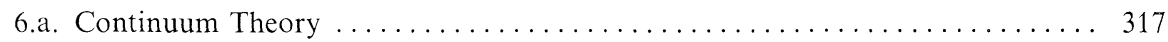

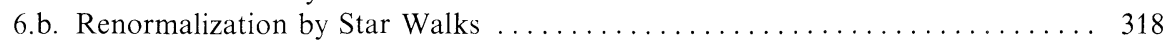

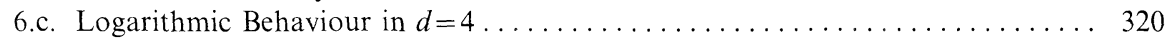

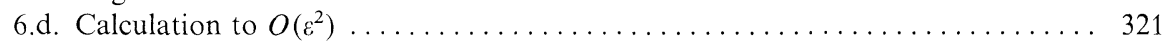

6.e. Three-Walk Crossings ....................................... 324

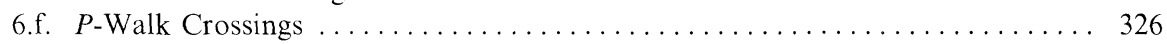

\section{Introduction}

Intersection properties of random walks or Brownian paths have been a long standing problem, starting from the works by Dvoretzky et al. [1], and Erdös and Taylor [2,3]. In particular, it is known [4] that two infinite (discrete) random walks in $\mathbb{Z}^{4}$ have a non-empty set of common points, while [1] two infinite Brownian (continuous) paths do not intersect in $\mathbb{R}^{4}$. For a space dimension $d \geqq 5$ the intersection sets are empty in both cases [1-5]. In this sense $d=4$ is a critical value for the intersection properties of two independent walks or paths, and this is wellknown to be intimately related to the non-triviality of the $\phi_{d}^{4}$ field theory [6-10] for $d$ $<4$, and to the theory of critical phenomena, where critical exponents take nonmean field values for $d<4$. For the statistical physicist, the above remark of the nonequivalence of intersections of discrete walks in $\mathbb{Z}^{4}$ and of continuous Brownian paths in $\mathbb{R}^{4}$ is entirely reminiscent of the existence of logarithmic corrections in a $\phi_{4}^{4}$ theory with an ultraviolet cut-off (the lattice spacing of $\mathbb{Z}^{4}$ ), while the continuum $\phi_{4}^{4}$ theory is widely believed to be trivial.

Recently, Lawler [11-13] has been able to give, by rather detailed probabilistic methods, logarithmic bounds on the probability of intersections of two random walks in four dimensions $[11,12]$, and of three random walks in three dimensions [13]. He considered two trajectories in $\mathbb{Z}^{4} \Pi_{1}(0, n)$ and $\Pi_{2}(0, n)$ of two simple random walks of $n$ steps, starting at $\mathbf{0}$ and $\mathbf{x}_{0}$ respectively, and their probability of no intersection after $n$ steps

$$
p(n)=P\left\{\Pi_{1}(0, n) \cap \Pi_{2}(0, n)=\emptyset\right\} .
$$

The results are for $n$ large

$$
p(n) \approx 1-a(\ln n)^{-1}
$$

if $\left|x_{0}\right|^{2} \approx n$, with a prefactor $a$ of the logarithm depending on $\left|x_{0}\right|^{2} / n=\alpha$, and for $\mathbf{x}_{0}=\mathbf{0}$

$$
p(n) \approx(\ln n)^{-1 / 2} .
$$


Actually (1.3) is only asserted to be the actual decay rate of $p(n)$, logarithmic bounds being obtained, which do not suffice to prove rigorously (1.3) as asymptotic limit. More precisely [11] it was shown that

$$
c(\ln n)^{-1} \leqq p(n) \leqq c^{\prime}(\ln n)^{-1 / 2},
$$

where $c$ and $c^{\prime}$ are constants, and [12] that $\lim _{n \rightarrow \infty}(\ln n)^{r} p(n)=\infty$ for all $r>1 / 2$. Similar bounds as in (1.3bis) have been rederived by Felder and Fröhlich [10] using (rigorous) methods in field theory, (see also Aizenman [9]). One should note also that more recently a different probabilistic approach, using "intersection local times", has been devised [14-17] for studying the intersection properties of random walks or Brownian paths. In particular [15], conjecture (1.3) appears likely in this approach. Lawler [13] has also considered the triple intersections in $\mathbb{Z}^{3}$ of three simple random walks, $d=3$ being there the upper critical dimension. The results for three trajectories $\Pi_{i}(0, n)$ are

1. For two walks starting at 0 , and a third starting at a distance $x_{0} \approx \sqrt{n}$,

$$
P\left\{\Pi_{1}(0, n) \cap \Pi_{2}(0, \infty) \cap \Pi_{3}(0, \infty) \neq \emptyset\right\} \approx(\ln n)^{-1} .
$$

2. For three walks starting at the origin

$$
P\left\{\Pi_{1}(0, n) \cap \Pi_{2}[0, \infty) \cap \Pi_{3}[0, \infty)=\emptyset\right\} \approx(\ln n)^{-1 / 4} .
$$

Actually, (1.4) is proven by upper and lower bounds, while the $(\ln n)^{-1 / 4}$ decay rate of (1.5) is rigorously obtained as an upper bound, the lower being only conjectured. Lawler [11,13] also presents results for a variety of other probabilities of the same type, like those of two-sided walks, to be described below.

The aim of the present article is to propose a different approach, which embodies both analytical calculations on Brownian motions (like in the probabilistic approaches) and renormalization (like in field theory), but directly applied to the Brownian intersection theory. This approach comes from polymer physics [18-20]. It has to be adapted to treat intersection properties of random walks. Polymers indeed correspond to random walks which are both self- and mutually avoiding [18-21]. Here only the mutual avoidance properties are relevant. This case also exists in physics and corresponds to polymer solutions with selective interactions or "chemical mismatch" [22]. We use for that purpose a continuum model, derived from the standard Edwards' model for polymers, and which describes the intersections of, e. g., two independent Brownian processes in $\mathbb{R}^{d}$, for $d=4-\varepsilon, \varepsilon \geqq 0$. Using renormalization theory, we are able to reach the limit $\varepsilon=0, d=4$. We then recover and extend Lawler's results. For instance, for two walks starting at $\mathbf{0}$ and $\mathbf{x}_{0}$, we obtain in four dimensions the universal limit

$$
\lim _{n \rightarrow \infty} 2 \ln n P\left\{\Pi_{1}(0, n) \cap \Pi_{2}(0, n) \neq \emptyset\right\}=\frac{1}{\alpha}\left(1-e^{-\alpha}\right)-E_{i}(-\alpha),
$$

where $\alpha=\lim _{n \rightarrow \infty}\left|x_{0}\right|^{2} / 2 n$, and where $E_{i}(-\alpha)$ is the exponential-integral function

$$
E_{i}(-\alpha)=-\int_{\alpha}^{\infty} \frac{e^{-t}}{t} d t
$$


The finite limiting value actually corrects one $(\ln (1+1 / \alpha))$ asserted by Lawler in [11]. For three walks $(0, n)(0, \infty),(0, \infty)$ starting respectively at $\left(\mathbf{x}_{0}, \mathbf{0}, \mathbf{0}\right)$, we find similarily in $\mathbb{Z}^{3}$ the new result

$$
\begin{aligned}
\lim _{n \rightarrow \infty} & 4 \ln n P\left\{\Pi_{1}(0, n) \cap \Pi_{2}(0, \infty) \cap \Pi_{3}(0, \infty) \neq \emptyset\right\} \\
= & \int_{0}^{1} d x(1-\sqrt{1-x}) e^{-\alpha x} \frac{d x}{x}-E_{i}(-\alpha)
\end{aligned}
$$

which gives the exact universal form of (1.4). By the same direct method, we reobtain (1.3)(1.5) for walks all starting at the same point.

We also present a series of generalizations. A first generalization consists in looking at the intersections of $P$ paths $(P \geqq 2)$ at the same point, the intersections of $p$ paths $1 \leqq p \leqq P-1$, being not considered. These $P$-body intersections occur (in probability) only below a critical (continuous) dimension $d^{*}=2 P /(P-1)$. We show how to calculate the scaling behaviour of the probabilities of multiple intersections of $P$ walks in $\mathbb{R}^{d}$, for $d \leqq d^{*}$, including their universal logarithmic behaviour in $\mathbb{R}^{d^{*}}$, which generalizes $(1.3)(1.5)$.

A second progress in the known results is obtained for the standard intersections of two walks $(P=2)$, by calculating the scaling behaviour of $p(n)(1.3)$ for $d<4$

$$
p(n) \approx n^{\zeta}, \quad n \rightarrow \infty,
$$

where $\zeta$ is an universal critical exponent which depends only on the space dimension $2 \leqq d<4$. We calculate it to $O\left(\varepsilon^{2}\right)$, where $\varepsilon=4-d$. This second order (two-loop) calculation allows also [20] in $d=4$ the obtention of all the subdominant $O(\ln \ln n / \ln n)$ universal $\operatorname{logarithmic}$ correction terms in asymptotic results like (1.2)-(1.7).

Finally, rather than considering only two (or $P$ ) walks starting at the same point, one can discuss nets of random walks in $\mathbb{Z}^{d}$, or Brownian paths in $\mathbb{R}^{d}$, for any topology. A net $\mathscr{G}$ is determined by requiring that a certain number of independent walks of lengths $n$ meet at some prescribed vertices, where two, three, $\ldots, L, \ldots$ walks meet. The vertices are not fixed in space, but the topology of the network, i.e. the net of walks, although arbitrary, is fixed. In other words, the topography of the walks is similar to that of a hydrographical network made of rivers flowing ones into the others at the same prescribed confluence points. Other crossings of the rivers are forbidden. We calculate in this work the probability $P(\mathscr{G})$ that the walks have no other intersection points than the prescribed confluence vertices. One can forbid double points in $d \leqq 4$, or only triple points in $d \leqq 3$, or $\ldots p$-tuple points in $d \leqq d^{*}$, and obtain each time a different universal scaling behaviour, which is evaluated here as an explicit function of the topology of the network. We find, below the critical dimension, that the probability $P(\mathscr{G})$ scales like

$$
P(\mathscr{G}) \approx n^{-\frac{d}{2} \mathscr{L}+\zeta_{\mathscr{G}}}, \quad n \rightarrow \infty,
$$

where $\mathscr{L}$ is the number of independent loops in $\mathscr{G}$, and where $\zeta_{\mathscr{G}}$ is a new universal exponent, topology dependent. We calculate $\zeta_{\mathscr{G}}$ to order $O\left(\varepsilon^{2}\right), \varepsilon=4-d$, when twowalk intersections are excluded, and to order $O\left(\varepsilon^{\prime}\right), \varepsilon^{\prime}=3-d$, when only three-walk 
intersections are excluded. We generalize this to $P$ walks, with $\varepsilon=\frac{2 P}{P-1}-d(>0)$. At the critical dimensions, $P(\mathscr{G})$ scales like

$$
P(\mathscr{G}) \approx n^{-\frac{d}{2} \mathscr{L}}(\ln n)^{3 *}, \quad n \rightarrow \infty,
$$

where $3_{\mathscr{G}}$ is a new universal logarithmic exponent, generalizing $-\frac{1}{2}$ or $-\frac{1}{4}$ in (1.3), (1.5). We calculate explicitly $\mathcal{Z}_{\mathscr{G}}$ as a function of the topology of $\mathscr{G}$, in the cases where two-walk, three-walk, ...P-walk intersections are excluded.

The continuum model that we use, for instance in the case of the intersections of two independent Brownian paths in $\mathbb{R}^{d}$, is given by the probability weight

$$
\begin{aligned}
\mathscr{P}\left\{\mathbf{r}_{1}, \mathbf{r}_{2}\right\}= & \exp \left\{-\frac{1}{2} \int_{0}^{s_{1}}\left(\frac{d \mathbf{r}_{1}}{d s_{1}}\left(s_{1}\right)\right)^{2} d s_{1}-\frac{1}{2} \int_{0}^{s_{2}}\left(\frac{d \mathbf{r}_{2}}{d s_{2}}\left(s_{2}\right)\right)^{2} d s_{2}\right. \\
& \left.-b \int_{0}^{s_{1}} d s_{1} \int_{0}^{s_{2}} d s_{2} \delta^{d}\left[\mathbf{r}_{1}\left(s_{1}\right)-\mathbf{r}_{2}\left(s_{2}\right)\right]\right\},
\end{aligned}
$$

where $\mathbf{r}_{1}\left(s_{1}\right), 0 \leqq s_{1} \leqq S_{1} ; \mathbf{r}_{2}\left(s_{2}\right), 0 \leqq s_{2} \leqq S_{2}$ are two Brownian trajectories in $\mathbb{R}^{d}$, interacting via a local repulsive distribution $\delta^{d}$, with an interaction coefficient $b>0$. For $b \rightarrow \infty$, (1.9) describes mutually avoiding walks. We work in dimensional regularization, which amounts to continue analytically the theory from $d<2$ towards $d=4$. For $d<2$ no divergences appear in the perturbation expansion of the model, while they occur at some poles for $2 \leqq d<4$. All quantities are calculated as meromorphic functions of $d$. The limit $S_{1}, S_{2} \rightarrow \infty$ (actually equivalent to $b \rightarrow \infty$, see below) will yield the universal properties of the intersections of long Brownian paths in $d \leqq 4$. It is also to be noted that working in dimensional regularization with $d=4$ $-\varepsilon, \varepsilon>0$, allows one to take the $d=4$ limit, after renormalization. Then one obtains automatically the scaling limit of the theory with an ultraviolet cut-off in four dimensions, as discussed in detail in ref. [20].

Let us briefly discuss the relation of this approach (1.9) to the field theoretic representation of polymer problems [21,23,24,5-10]. To each random walk $\beta(=1,2)$ is associated a field $\phi^{\beta}(x)$ with $n$ components $\phi_{i}^{\beta}(x) i=1, \ldots, n$, where $x$ is a point of the lattice $\mathbb{Z}^{d}$. The local repulsive interaction between walks is simulated by a field interaction term $[5-10] b \sum_{x \in \mathbb{Z}^{d}} \sum_{\beta \neq \gamma}\left|\phi^{\beta}(x)\right|^{2}\left|\phi^{\gamma}(x)\right|^{2}$ in the Lagrangian. A mass term $m^{2} \sum_{\beta}\left|\phi^{\beta}(x)\right|^{2}$ corresponds to the exponential killing factor $e^{-m^{2} \sum_{\beta} S_{\beta}}$ of the times $\left\{S_{\beta}\right\}$ allowed to the set of walks $\{\beta\}$ in (1.9). Then the $n \rightarrow 0$ limit [23-25] of the Euclidean field theory corresponds to the theory (1.9) of Brownian paths with a mutual local repulsive interaction between any two paths. This correspondence, implicit in Symanzik's representation of field theory [26], has been fully understood and exploited in polymer physics, starting from the original works by de Gennes [23] and des Cloizeaux [24], and using Wilson's theory of critical phenomena [27]. More recently, this correspondence has also been quite fruitful in mathematical physics, by mixing random walks and field theories [5-10], yielding rigorous results in the latter. But here we want to follow the other direction [18-20,22], without employing field theory, but only direct renormalization methods, which deal directly with locally interacting polymers, or, as in (1.9), with locally interacting 
Brownian paths. In these direct theories one can perform any analytic calculation of configurations of random walks with some specific intersections. Hence, the parameters are directly those of interest for the probabilist mathematician. In particular, what is called "local time" [14-17] corresponds precisely to the interaction integral in Edwards' formulation (1.9) [28-32] of interacting random chains. [The existence of weight (1.9) could be established as in Westwater [29-32] who studied the standard Edwards' model [28]]. Admittedly, up to now, the validity of the direct renormalization methods for the Edwards' model has only been established [33-35] by a Laplace-de Gennes transformation [25] into a $O(n)$ field theory in the limit $n \rightarrow 0$, and using the renormalization scheme of the field theory. So it has the same heuristic validity as the standard perturbative Callan-Symanzik renormalization of, e.g., the $\left(\varphi^{4}\right)_{d}$ field theory, as used by $\mathrm{K}$. Wilson for describing critical phenomena in $d=4-\varepsilon$. A similar derivation of the multiplicative renormalization structure could be performed for model (1.9), starting from the interacting field theory $\left\{\phi^{\beta}(x)\right\}$ described above. But the direct renormalization method is quite powerful and simple. New results and new generalizations to more random walks with various topologies (like those of star-walks, or nets of walks) are given in $\mathbb{R}^{d}, d \leqq 4$. We also consider in detail the case of triple intersections in $\mathbb{R}^{d}$, $d \leqq 3$, for various topologies, and of $P$-tuple intersections, using the same method.

We hope that this study could interest various readerships, by bridging a gap between probabilistic approaches and field theoretic ones. It can be used also as an heuristic mean to invent new exact results, which then remain to be proven rigorously, in the mathematician's sense. It could also suggest in probability theory more direct approaches to intersection properties, which should embody the salient features of renormalization theory.

\section{Intersections of Two Walks Near Four Dimensions}

\section{2.a. The Continuum Theory}

Let us consider first the simplest situation of two independent random walks of the same length. We describe them by a continuum theory in $\mathbb{R}^{d}$ with $d<4$. At the end, we let $d \rightarrow 4$. The probability weight of configurations $\mathbf{r}_{1}(s), \mathbf{r}_{2}(s)$ in $\mathbb{R}^{d}$ is of the Edwards' type (1.9)

$$
\begin{aligned}
\mathscr{P}\left\{\mathbf{r}_{1}, \mathbf{r}_{2}\right\}= & \exp \left\{-\frac{1}{2} \int_{0}^{s} d s \dot{\mathbf{r}}_{1}^{2}(s)-\frac{1}{2} \int_{0}^{s} d s^{\prime} \dot{\mathbf{r}}_{2}^{2}\left(s^{\prime}\right)\right. \\
& \left.-b \int_{0}^{S} d s \int_{0}^{s} d s^{\prime} \delta^{d}\left[\mathbf{r}_{1}(s)-\mathbf{r}_{2}\left(s^{\prime}\right)\right]\right\} .
\end{aligned}
$$

$S$ is the "length" of the two walks, and by dimensional analysis one checks that it has the dimension of an area in the continuum theory. More precisely [18], for a single isolated random walk

$$
\left\langle[\mathbf{r}(S)-\mathbf{r}(0)]^{2}\right\rangle_{0}=d S,
$$

where the average is taken with the Brownian weight (2.1) for one walk $\left(b \delta^{d}\right.$ drops away). Thus $S$ replaces the usual number $n$ of steps in a lattice walk with $n / d \rightarrow S$. Let 
us then perform the rescaling $\mathbf{r}_{i}=S^{1 / 2} \varrho_{i}, i=1,2$, and $s=S x, \varrho$ and $x$ being now dimensionless. We get for (2.1)

$$
\begin{aligned}
\mathscr{P}\left\{\varrho_{1}, \varrho_{2}\right\}= & \exp \left\{-\frac{1}{2} \int_{0}^{1} d x \dot{\underline{\Theta}}_{1}^{2}(x)-\frac{1}{2} \int_{0}^{1} d x^{\prime} \dot{\varrho}_{2}^{2}\left(x^{\prime}\right)\right. \\
& \left.-(2 \pi)^{d / 2} z \int_{0}^{1} d x \int_{0}^{1} d x^{\prime} \delta^{d}\left[\varrho_{1}(x)-\varrho_{2}\left(x^{\prime}\right)\right]\right\},
\end{aligned}
$$

where the dimensionless parameter $z$ of the two-point interaction is defined as [18]

$$
z=(2 \pi)^{-d / 2} b S^{2-d / 2} .
$$

Clearly, for $d>4, z \rightarrow 0$ for $S \rightarrow \infty$ and the intersection local time [14-17, 36, 37]

$$
\int_{0}^{S} d s \int_{0}^{S} d s^{\prime} \delta^{d}\left[\mathbf{r}_{1}(s)-\mathbf{r}_{2}\left(s^{\prime}\right)\right] \sim S^{2-d / 2}
$$

is irrelevant (vanishing). On the contrary, for $d<4, z \rightarrow \infty$ when $S \rightarrow \infty$, and the intersections are important. As is well known, $d=4$ is the marginal case, where logarithmic behaviour occurs. In the limit $z \rightarrow \infty$, the weight (2.1) (2.1 bis) selects only configurations without crossings, and enables us to discuss intersection probabilities. For doing this, we define the averages with respect to weight (2.1), as the functional integrals

$$
\langle\ldots\rangle=\frac{\int d\left\{\mathbf{r}_{1}\right\} d\left\{\mathbf{r}_{2}\right\} \mathscr{P}\left\{\mathbf{r}_{1}, \mathbf{r}_{2}\right\}(\ldots)}{\int d\left\{\mathbf{r}_{1}\right\} d\left\{\mathbf{r}_{2}\right\} \delta^{d}\left(\mathbf{r}_{1}(0)\right) \delta^{d}\left(\mathbf{r}_{2}(0)\right) \mathscr{P}_{0}\left\{\mathbf{r}_{1}\right\} \mathscr{P}_{0}\left\{\mathbf{r}_{2}\right\}},
$$

where $\mathscr{P}_{0}$ is the pure Brownian weight of the trajectories for $b=0$, which defines the normalization in the continuum theory. Following Lawler's notation [11, 12], let us consider the probabilitity that two simple random walks in $\mathbb{Z}^{d}$, starting from 0 (Fig. 1) do not intersect again before step $n: P\left\{\Pi_{1}[0, n) \cap \Pi_{2}(0, n)=\emptyset\right\}$. In our continuum theory this is just $\mathscr{Z}(S, S ; \mathbf{0}, \mathbf{0})$, where

$$
\mathscr{Z}(S, S ; \mathbf{x}, \mathbf{y})=\left\langle\delta^{d}\left(\mathbf{r}_{1}(0)-\mathbf{x}\right) \delta^{d}\left(\mathbf{r}_{2}(0)-\mathbf{y}\right)\right\rangle
$$

is the correlator of two walks of lengths $S$ starting at $\mathbf{x}$ and $\mathbf{y}$ in $\mathbb{R}^{d}$. Owing to (2.5), $\mathscr{Z}$ is dimensionless. The critical limit $z \rightarrow \infty$ will yield the continuum analogue of the no-intersection probability $P$ above.

\section{2.b. Diagrammatic Rules}

The strategy is the following. We first calculate $(2.5),(2.6)$ by perturbation theory of weight $\mathscr{P}(2.1)$ in powers of $b$. To first order, we have

$$
\mathscr{Z}(S, S ; \mathbf{0}, \mathbf{0})=1-b \int_{0}^{S} d s \int_{0}^{S} d s^{\prime}\left\langle\delta^{d}\left[\mathbf{r}_{1}(s)-\mathbf{r}_{2}\left(s^{\prime}\right)\right] \delta^{d}\left[\mathbf{r}_{1}(0)\right] \delta^{d}\left[\mathbf{r}_{2}(0)\right]\right\rangle_{0}+O\left(b^{2}\right),
$$

where the subscript 0 stands for the Brownian weight with $b=0$. (We see that at this $\operatorname{order} \mathscr{Z}$ is given by the average value of the intersection local time (2.4) but at higher orders correlations of local times come in.) Such a term (2.7) is represented by a 

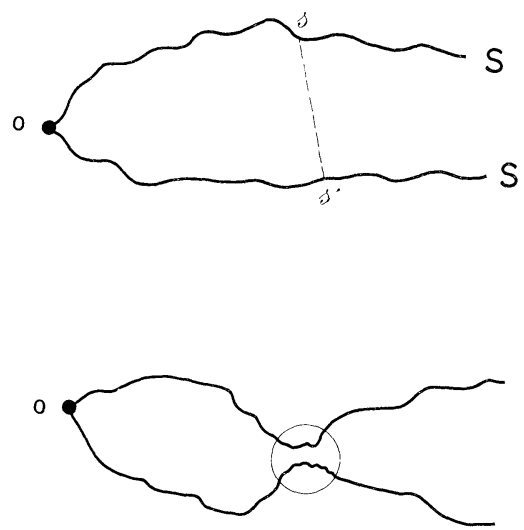

Fig. 1. One-loop diagram contributing to the probability of no further intersection of two independent random walks starting at the origin 0 . The dotted line represents conventionally a local contact $-b \delta^{d}\left[\mathbf{r}_{1}(s)-\mathbf{r}_{2}\left(s^{\prime}\right)\right]$ in space, between points of abscissa $s$ and $s^{\prime}$

diagram [18] as in Fig. 1 with a dotted line for the $\delta^{d}$ contact. Calculations are always performed by Fourier transforming [18-20]

$$
\delta^{d}(\mathbf{r})=(2 \pi)^{-d} \int d^{d} q e^{i \mathbf{q} \cdot \mathbf{r}},
$$

and using for each pure $(b=0)$ Brownian path $\mathbf{r}$ the Green function

$$
\left\langle e^{i \mathbf{q} \cdot[\mathbf{r}(s)-\mathbf{r}(0)]}\right\rangle_{0}=e^{-\frac{1}{2} q^{2} s} .
$$

Hence we trivially find for (2.7)

$$
\mathscr{Z}(S, S ; \mathbf{0}, \mathbf{0})=1-b \int \frac{d^{d} q}{(2 \pi)^{d}} \int_{0}^{S} d s \int_{0}^{S} d s^{\prime} e^{-\frac{1}{2} q^{2}\left(s+s^{\prime}\right)} .
$$

This is a particular case of general diagrammatic rules [18] for calculating an average like (2.5) in perturbation series of $b$. They are easily obtained by expanding the exponential in (2.1). These rules, which we shall need all along, are the following (Fig. 2).

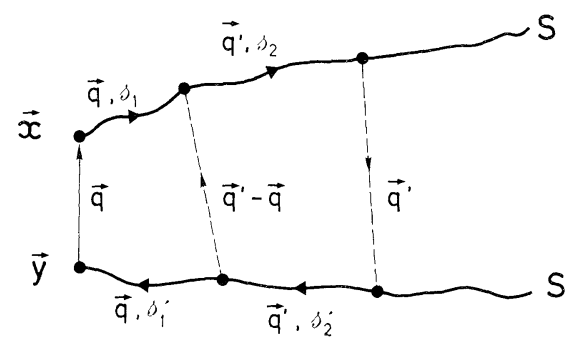

Fig. 2. A second order (two-loop) diagram contributing to $\mathscr{Z}(S, S, \mathbf{x}, \mathbf{y})$ [Eq. (2.6)], and the associated integration variables appearing in Eq. (2.11), as an illustration of the diagrammatic rules of Sect. (2.b) 
1. Diagrams are made of walks (continuous lines) interacting via arbitrary dotted lines joining two interaction points on walks, with a factor $(-b)$ for each interaction.

2. When some physical points on the walks are at some prescribed positions $\{\mathbf{x}, \mathbf{y}, \ldots\}$ in $\mathbb{R}^{d}$ one draws also a set of independent vectors $\{\mathbf{x}-\mathbf{y}, \ldots\}$ which span the independent relative positions.

3. A set of independent loops is selected in each interaction diagram, which involve both continuous walk lines, interaction dotted lines, and relative position vectors. An independent momentum $\mathbf{q}$ flows along each loop, with integration measure $\int \frac{d^{d} q}{(2 \pi)^{d}}$.

4. Each of the relative vectors $\{\mathbf{x}-\mathbf{y}, \ldots\}$ of rule 2 carries a momentum $\Sigma \mathbf{q}$ which is the sum of the momenta of the independent loops to which the vector belongs. Then it contributes a factor $e^{i(\mathbf{x}-\mathbf{y}) \cdot \Sigma \mathbf{q}}$ to the integrand.

5. Along each segment of length $s$ of a walk, determined by two successive interaction points along a same walk, one evaluates the total momentum flowing along it, which is the algebraic sum $\Sigma \mathbf{q}$ of the momenta of all the independent loops to which the segment belongs. This segment contributes then a factor $e^{-\frac{1}{2}(\Sigma \boldsymbol{q})^{2} s}$ to the integrand.

6. One integrates over all independent momenta, and all positions of interaction points which preserve the topology of the diagram.

7. One sums over non-topologically equivalent diagrams.

Equation (2.10) corresponding to Fig. 1 gives a first trivial example. The contribution of the second order diagram of Fig. 2 to $\mathscr{Z}(S, S ; \mathbf{x}, \mathbf{y})(2.6)$ is for instance

$$
\begin{aligned}
& (-b)^{2} \int \frac{d^{d} q}{(2 \pi)^{d}} \frac{d^{d} q^{\prime}}{(2 \pi)^{d}} \int_{0}^{\infty} d s_{1} \int_{0}^{\infty} d s_{2} \theta\left(S-s_{1}-s_{2}\right) \\
& \cdot \int_{0}^{\infty} d s_{1}^{\prime} \int_{0}^{\infty} d s_{2}^{\prime} \theta\left(S-s_{1}^{\prime}-s_{2}^{\prime}\right) e^{i \mathbf{q} \cdot(\mathbf{x}-\mathbf{y})} e^{-\frac{1}{2} q^{2}\left(s_{1}+s_{1}^{\prime}\right)-\frac{1}{2} q^{\prime 2}\left(s_{2}+s_{2}^{\prime}\right)} .
\end{aligned}
$$

Now, for calculating the contribution of a diagram obtained by the above rules, one integrates first onto the $L$ Gaussian loop variables $\mathbf{q}_{l}, l=1, \ldots, L$, with the wellknown Gaussian formula

$$
\begin{aligned}
& \int \prod_{l=1}^{L} d^{d} q_{l} \exp \left(-\frac{1}{2} \sum_{l, l^{\prime}} \mathbf{q}_{l} M_{l l^{\prime}} \mathbf{q}_{l^{\prime}}+i \sum_{l} \mathbf{q}_{l} \cdot \mathbf{x}_{l}\right) \\
& \quad=(2 \pi)^{\frac{d}{2}}(\operatorname{det} M)^{-d / 2} \exp \left(-\frac{1}{2} \sum_{l} \mathbf{x}_{l}\left(M^{-1}\right)_{l l^{\prime}} \mathbf{x}_{l^{\prime}}\right),
\end{aligned}
$$

where the matrix $M_{l l^{\prime}}$ acts here only on the loop indices $l=1, \ldots, L$, which are decoupled from the $\mathbb{R}^{d}$ space components. Clearly (2.12) now allows the analytic continuation [38] of the theory to non-integer values of $d$. [One should also note that after Gaussian integration over all momenta q's one gets an integrand in terms of parameters $s$, which is exactly that of the Schwinger-Feynman " $\alpha$ parameter" representation of the $\left(\varphi^{2}\right)_{d}^{2}$ quantum field theory. The only difference lies in the domain of integration. The walk parameters $\{s\}$ are bounded by constraints on the 
lengths $S$ of the various walks, while in usual QFT the $\alpha$ parameters, $\alpha \in[0, \infty[$, are exponentially killed by mass terms $e^{-m^{2} \Sigma \alpha}$. Of course, multiple Laplace transforms relate both cases.]

Let us now return to the evaluation of $\mathscr{Z}(2.10)$. We have after Gaussian integration

$$
\begin{aligned}
\mathscr{Z} & =1-b(2 \pi)^{-d / 2} \int_{0}^{S} d s \int_{0}^{S} d s^{\prime}\left(s+s^{\prime}\right)^{-d / 2} \\
& =1-z \frac{1}{(1-d / 2)(2-d / 2)}\left[2^{2-d / 2}-2\right] .
\end{aligned}
$$

This quantity diverges for $d=4$, and for $d=4-\varepsilon$ the leading diverging order in $1 / \varepsilon$ reads

$$
\mathscr{Z}=1-z\left[\frac{2}{\varepsilon}+O(1)\right]+O\left(z^{2}\right)
$$

Of course, one could wonder why such an expansion is useful. First, the expansion parameter $z$ grows without limit, second, the coefficients of the expansion have poles in $\varepsilon$ when $\varepsilon \rightarrow 0$. (To order $z^{p}$, the pole is of order $\frac{1}{\varepsilon^{p}}$.) That is where renormalization, which exploits the mathematical structure of this double TaylorLaurent expansion, comes in.

\section{2.c. Renormalization}

Briefly stated, renormalization [18-20,27,33-35] is a way to eliminate poles in $1 / \varepsilon$ by a suitable change to "renormalized" variables. In the present case of intersections of independent random walks, we have to adapt the direct renormalization method devised originally for standard polymers [18-20,22]. The idea is to substitute to the interaction parameter $z(2.3)$ in the scaling functions a function $g(z, \varepsilon)$ which converges to a finite fixed point limit $g^{*}$ when $z \rightarrow \infty$, and for $d<4$. A scaling function is here a function of $z, \varepsilon$, which still has poles of all orders in $\varepsilon$, but which reaches a fixed point limit for $z \rightarrow \infty$, (for $d<4$ ). For instance, here, we expect [10] the probability $\mathscr{Z}$ above to scale for $S$ large (and $d<4$ ) like $\mathscr{Z} \sim S^{\zeta}$, where $\zeta$ is a universal critical exponent. Hence the scaling function $\left.\zeta(z, \varepsilon) \equiv S \frac{\partial}{\partial S} \ln \mathscr{Z}\right|_{b, \varepsilon}=\left.\frac{\varepsilon}{2} z \frac{\partial}{\partial z} \ln \mathscr{Z}\right|_{\varepsilon}$ should reach the fixed point limit $\zeta$ when $z \rightarrow \infty$. Then the renormalization principle $[18-20,33,34]$ is that a (singular) TaylorLaurent expansion $g(z, \varepsilon)$ exists such that the substitution of $g$ to $z$ in any scaling function must give a new double series in $g, \varepsilon$, which is regular to all orders in $g$ when $\varepsilon \rightarrow 0$.

A possibility for $g$ is furnished [18] by the dimensionless "second virial coefficient" of two independent random walks, here defined as

$$
g=\mathscr{Z}_{2}(S, S)(2 \pi S)^{-d / 2},
$$


where $\mathscr{Z}_{2}(S, S)$ is the connected partition function of two walks interacting via weight (2.1):

$$
\mathscr{Z}_{2}(S, S)=b\left\langle\int_{0}^{S} d s_{1} \int_{0}^{S} d s_{2} \delta^{d}\left[\mathbf{r}_{1}\left(s_{1}\right)-\mathbf{r}_{2}\left(s_{2}\right)\right] \delta^{d}\left[\mathbf{r}_{1}(0)\right]\right\rangle,
$$

i.e. the two walks have at least one point in common. (In the $z \rightarrow \infty$ limit, they will have only this point in common). A probabilistic meaning can be given to the dimensionless quantity $g$ (2.16), (2.17): it is the average intersection local time of two mutually-avoiding Brownian paths intersecting once [normalized by $\left.(2 \pi S)^{-d / 2}\right]$. Note that $z(2.3)$ can be written similarly as

$$
z=b(2 \pi S)^{-d / 2}\left\langle\int_{0}^{S} d s_{1} \int_{0}^{S} d s_{2} \delta^{d}\left[\mathbf{r}_{1}\left(s_{1}\right)-\mathbf{r}_{2}\left(s_{2}\right)\right] \delta^{d}\left[\mathbf{r}_{1}(0)\right]\right\rangle_{0},
$$

where the only change with respect to (2.17) is the occurrence of the pure Brownian average $\langle\ldots\rangle_{0}$ instead of that of two mutually avoiding paths $\langle\ldots\rangle$. So one basic statement of renormalization theory is that the expansion of scaling functions (like $\zeta(z, \varepsilon)$ above) is $\varepsilon$-regular in terms of the true intersection local time $g$ of two mutually avoiding Brownian paths, while it is singular in terms of the intersection local time $z$ of two simple Brownian paths. It is worth noting that $g$ reaches in the long time limit of the paths an universal fixed point limit (see below), which it would be interesting to study in probability theory.

Remark. Usually, the renormalization in QFT involves a multiplicative renormalization scheme, with renormalization conditions [27]. We have also here a multiplicative renormalization structure of the walk partition functions which will appear only later (Sect. 6), in more complicated situations involving nets of walks meeting at some branching points. We shall state there that the ratios of any partition function of complicated multiple walks to those of simple "star walks" reach finite limits. This multiplicative aspect of renormalization does not appear here since $\mathscr{Z}(S, S, \mathbf{x}, \mathbf{y})(2.10)$ for two mutually-avoiding paths is the simplest object of the theory, the (normalized) partition function of a single pure Brownian path being trivially one.

The first order diagrams contributing to $\mathscr{Z}_{2}$ to orders $b, b^{2}$ are shown in Fig. 3.
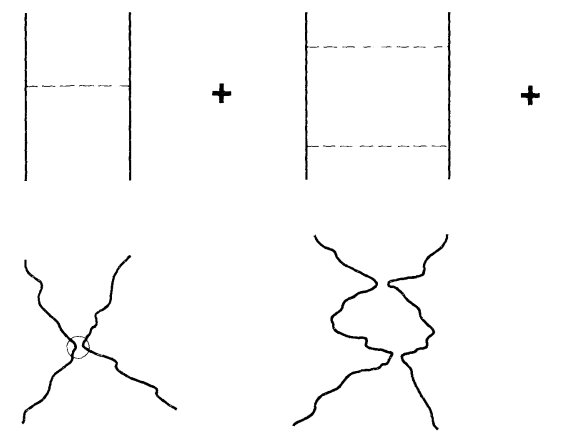
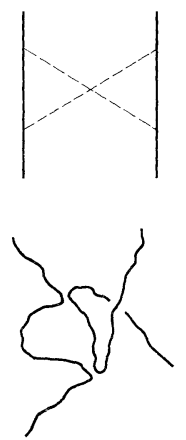

Fig. 3. The diagrams contributing to the connected partition function $\mathscr{Z}_{2}(S, S)(2.17)$ of two independent Brownian paths, and the actual conformations in direct space 
Their contributions are easily obtained from the diagrammatic rules above

$$
\begin{aligned}
\mathscr{Z}_{2} & =b S^{2}-2 b^{2} \int \frac{d^{d} q}{(2 \pi)^{d}} \int_{0}^{S} d s(S-s) \int_{0}^{S} d s^{\prime}\left(S-s^{\prime}\right) e^{-\frac{q^{2}}{2}\left(s+s^{\prime}\right)} \\
& =b S^{2}-2 b^{2}(2 \pi)^{-d / 2} \int_{0}^{S} d s \int_{0}^{S} d s^{\prime}(S-s)\left(S-s^{\prime}\right)\left(s+s^{\prime}\right)^{-d / 2} .
\end{aligned}
$$

The integration leads for $d=4-\varepsilon$ to

$$
\mathscr{Z}_{2}=b S^{2}\left[1-z\left(\frac{4}{\varepsilon}+\ldots\right)\right] \text {. }
$$

Hence the dimensionless "second virial coefficient" $g$ (2.16) reads for two independent random walks

$$
g=z\left\{1-z\left[\frac{4}{\varepsilon}+O(1)\right]+O\left(z^{2}\right)\right\} .
$$

This quantity can be shown, by generalizing the arguments of ref. [33, 34], to have a fixed point value for $z \rightarrow \infty$. We obtain it in a standard way, by introducing the Wilson function

$$
W(z, \varepsilon)=\left.S \frac{\partial}{\partial S} g\right|_{b, \varepsilon}=\left.\frac{\varepsilon}{2} z \frac{\partial}{\partial z} g\right|_{\varepsilon}=\frac{\varepsilon}{2} z-4 z^{2}+\ldots .
$$

We know [18, 27, 33-35] that the substitution of $g$ to $z$ in $W$ makes $W[g, \varepsilon]$ finite to all orders in $g$ when $\varepsilon \rightarrow 0$. (Here, this seems a triviality but the next order, to be considered later, will exhibit this fundamental mechanism.) So we compute

$$
W[g, \varepsilon]=\frac{\varepsilon}{2} g-2 g^{2}+O\left(g^{3}\right)
$$

Since $g \rightarrow g^{*}$ when $S \rightarrow \infty$, i.e. $z \rightarrow \infty$, one has $S \frac{\partial}{\partial S} g \rightarrow 0$, and $g^{*}$ is thus found from $W\left[g^{*}, \varepsilon\right]=0$. Hence

$$
g^{*}=\frac{\varepsilon}{4}+O\left(\varepsilon^{2}\right)
$$

In four dimensions, the situation is slightly different [20]. We set $\varepsilon=0$ right into $W[g, \varepsilon](2.21)$, since the latter is regular at $d=4$. Hence

$$
\left.S \frac{\partial}{\partial S} g\right|_{\varepsilon=0}=W[g, 0]=-2 g^{2}+O\left(g^{3}\right) .
$$

Integrating gives immediately

$$
g=\frac{1}{2 \ln S / s_{0}}+O\left(\frac{\ln \ln S}{\ln ^{2} S}\right)
$$

where $s_{0}$ is an integration constant, which plays the role of the ultraviolet cut-off. It is the reminiscence in the continuum theory of the lattice spacing. From now on, we shall always drop away $s_{0}$, except when necessary ( $S$ is large). 
Now the limit for $S$ large of the probability $\mathscr{Z}$ of no intersection of two random walks starting at the origin, is evaluated by a simple "Callan-Symanzik" equation. As already mentioned, $\mathscr{Z}$ scales for $d<4$ like $\mathscr{Z} \sim S^{\zeta}, S \rightarrow \infty$, where $\zeta$ is a universal critical exponent. So $S \frac{\partial}{\partial S} \ln \mathscr{Z}$ will reach a finite limit $\zeta$ when $S \rightarrow \infty$. We have from (2.15),

$$
\zeta(z, \varepsilon)=\left.S \frac{\partial}{\partial S} \ln \mathscr{Z}\right|_{b, \varepsilon}=\frac{\varepsilon}{2} z \frac{\partial}{\partial z} \ln \mathscr{Z}=-z+O\left(z^{2}\right) .
$$

Again $\zeta[g, \varepsilon]$, when $g$ is substituted to $z$, is a regular double series in $g$ and $\varepsilon[18,33-$ 35]. Here trivially

$$
\zeta[g, \varepsilon]=-g+O\left(g^{2}\right) .
$$

In the limit of large walks, $S \rightarrow \infty, g \rightarrow g^{*}$ and

$$
\zeta=-\frac{\varepsilon}{4}+O\left(\varepsilon^{2}\right)
$$

for $d=4-\varepsilon$. For $d=4$ on the contrary, we use definition (2.24) and (2.25), (2.23), to get

$$
S \frac{\partial}{\partial S} \ln \mathscr{Z}=-g+\ldots=-\frac{1}{2 \ln S}+\ldots
$$

which is trivially integrated at dominant order into

$$
\mathscr{Z}=C(\ln S)^{-1 / 2} \text {. }
$$

$C$ is a constant, here depending [20] on $b$, which is thus model or lattice dependent, and not universal. Equation (2.26) is just Lawler's result (1.3), obtained here by a trivial one-loop calculation. It is worth noting that the method by Lawler in $d=4$ has deep connections with our techniques, even if renormalization for $d \leqq 4$ is not used there. Indeed he compares walks of length $n$ and $n(\ln n)^{-\beta}$, where $\beta>0$ is fixed (which is a kind of scale transformation), and states [12] that for two walks starting at the same point $p(n) \approx p\left[n(\ln n)^{-\beta}\right]\left(1-\frac{1}{2} \beta \frac{\ln \ln n}{\ln n}\right)$, where $p(n)$ is the discrete analogue of $\mathscr{Z}$ here. The limit $\beta \rightarrow 0$ of this equation yields the differential equation $n \partial \ln p(n) / \partial n=-1 /(2 \ln n)$ which is nothing but (2.26). Hence, a probabilistic result like (2.26) can be seen as a consequence of perturbation expansion followed by renormalization up to $d=4$. In the next section, we consider along the same lines a series of other interesting geometrical cases.

\section{2.d. Other Geometrical Cases}

Lawler [11-13] considered various other situations. We follow the introduction of [13] for a summary of definitions and statements. Let $T_{1}(n, \omega)$ be a simple random walk in $\mathbb{Z}^{4}$ starting at $\mathbf{x}_{0}$ and $T_{2}$ a "two-sided" random walk independent of $T_{1}$, starting at $\mathbf{0}$. (A "two-sided" walk is gotten by taking two independent simple random walks, $T_{2}^{+}, T_{2}^{-}$, and setting $T_{2}(n)=T_{2}^{+}(n), n \geqq 0$, and $T_{2}(n)=T_{2}^{-}(n)$ for 
$n \leqq 0$. Let $\Pi_{1}, \Pi_{2}$ denote the paths of the walks

$$
\begin{aligned}
& \Pi_{i}\left[n_{1}, n_{2}\right]=\left\{T_{i}(n, \omega): n_{1}<n<n_{2}\right\}, \\
& \Pi_{i}\left(n_{1}, n_{2}\right)=\left\{T_{i}(n, \omega): n_{1} \leqq n \leqq n_{2}\right\},
\end{aligned}
$$

and similarly for $\Pi_{i}\left[n_{1}, n_{2}\right)$ and $\Pi_{i}\left(n_{1}, n_{2}\right]$.

Let us first consider walks starting at the same point $\left(\mathbf{x}_{0}=\mathbf{0}\right)$ and define

$$
p(n)=P\left\{\Pi_{1}[0, n] \cap \Pi_{2}(0, n]=\emptyset\right\} .
$$

Then there exists [11] $c_{1}, c_{1}>0$ satisfying

$$
c_{1}(\ln n)^{-1} \leqq p(n) \leqq c_{2}(\ln n)^{-1 / 2},
$$

and for $r>1 / 2[12]$

$$
\lim _{n \rightarrow \infty}(\ln n)^{r} p(n)=\infty
$$

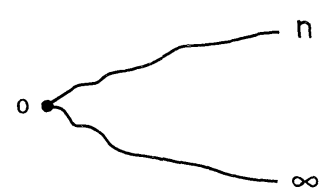

(a)

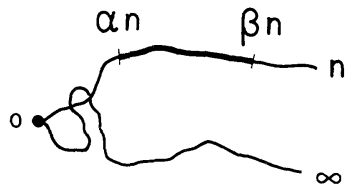

(c)

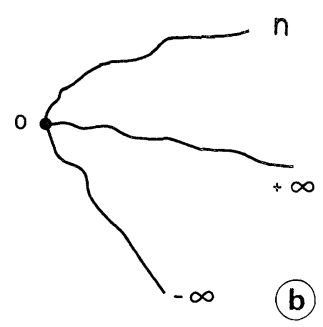

(b)

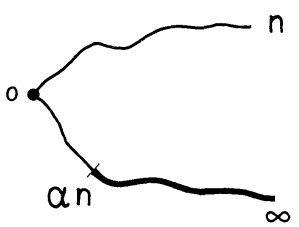

(d)

(e)

Fig. 4a-e. The different geometrical situations considered by Lawler. a The probability (2.29) that the trajectories of a random walk of length $n$ and of an infinite one, starting at the same point, do not cross anymore. $\mathrm{b}$ The same probability (2.30) that the finite walk trajectory does not cross a $[-\infty,+\infty]$ two-sided walk trajectory. c The probability (2.31) that the segment $(\alpha n, \beta n)$ does reintersect the trajectory of an infinite independent random walk. $d$ The half random walk $(\alpha n, \infty]$ does cross the walk $(0, n)$ [Eq. (2.32)]. e The probability that the two walks $(0, n)$ and $[0, \infty)$ starting at a distance $\left|\mathbf{x}_{0}\right| \approx \sqrt{n}$, intersect each other [Eq. (2.33)] 
Hence the actual decay rate should be $p(n) \approx(\ln n)^{-1 / 2}$, which we found above heuristically in (2.26). The same is true [11,12] for the no further intersection probability $q(n)$ of a finite walk and an infinite walk, in $\mathbb{Z}^{4}$, both starting at 0 (Fig. 4a)

$$
q(n)=P\left\{\Pi_{1}[0, n] \cap \Pi_{2}(0, \infty]=\emptyset\right\} \approx(\ln n)^{-1 / 2},
$$

which satisfies (2.27)(2.28). For the two-sided walk $T_{2}$ (Fig. 4b) one has

$$
P\left\{\Pi_{1}(0, n] \cap \Pi_{2}[-\infty,+\infty]=\emptyset\right\} \approx(\ln n)^{-1} .
$$

For the intersections of parts of walks in $\mathbb{Z}^{4}$ both starting at the origin (Fig. 4c), Lawler found also (Theorem 4.1 of [11]), for $0<\alpha<\beta<\infty$

$$
\lim _{n \rightarrow \infty}(\ln n) P\left\{\Pi_{1}(\alpha n, \beta n) \cap \Pi_{2}(0, \infty] \neq \emptyset\right\}=\frac{1}{2} \ln (\beta / \alpha),
$$

and for $0<\alpha<\infty$ (Fig. $4 \mathrm{~d}$ )

d)

$$
\lim _{n \rightarrow \infty}(\ln n) P\left\{\Pi_{1}(0, n) \cap \Pi_{2}(\alpha n, \infty] \neq \emptyset\right\}=\frac{1}{2} \ln (1+1 / \alpha) .
$$

Finally, for two walks $T_{1}, T_{2}$ starting at $\mathbf{0}$ and $\mathbf{x}_{0}$ respectively (Fig. 4e), supposing the limit $\alpha=\lim _{n \rightarrow \infty}\left|\mathbf{x}_{0}\right|^{2} / 2 n$ to exist, the statement is [11].

$$
\lim _{n \rightarrow \infty}(\ln n) P\left\{\Pi_{1}(0, n) \cap \Pi_{2}[0, \infty] \neq \emptyset\right\}=l(\alpha),
$$

where (incorrectly) $l(\alpha)=\frac{1}{2} \ln (1+1 / \alpha)$. As we shall see, the limit is rather given by (1.6). Let us now derive results (2.29) to (2.33) by direct renormalization.

Case a. $q(n)$ is, in our continuum model (2.1), obtained as the limit where one of the walks is infinite, of the correlator $(2.6) q(n) \rightarrow \mathscr{Z}(S, \infty ; \mathbf{0 , 0})$. To first order in $b$, (Fig. 5a), it reads from (2.13),

$$
\mathscr{Z}(S, \infty ; \mathbf{0}, \mathbf{0})=1-b(2 \pi)^{-d / 2} \int_{0}^{S} d s \int_{0}^{\infty} d s^{\prime}\left(s+s^{\prime}\right)^{-d / 2}=1+z \frac{1}{(1-d / 2)(2-d / 2)} .
$$

Its Laurent expansion in $1 / \varepsilon$ is thus

$$
\mathscr{Z}(S, \infty)=1-\frac{2 z}{\varepsilon}+\ldots,
$$

and is identical to that of $\mathscr{Z}(S, S ; \mathbf{0}, \mathbf{0})(2.15)$. Hence the analysis of Sect. 2.c applies entirely to $\mathscr{Z}(S, \infty)$ and leads to

$$
\begin{array}{rlrl}
q(n) \rightarrow \mathscr{Z}(S, \infty) & \sim(\ln S)^{-1 / 2}, & & d=4, \\
\mathscr{Z}(S, \infty) \sim S^{-\varepsilon / 4+\ldots}, & & d=4-\varepsilon .
\end{array}
$$

Actually, this identity holds to all orders in $\varepsilon$, the scaling properties of $\mathscr{Z}(S, S) \sim \mathscr{Z}(S, \infty) \sim S^{\zeta}$, being expected to be the same on physical grounds. It means that the steps of the infinite walk after step $S$ are irrelevant for the intersections with a finite walk of length $S$. 

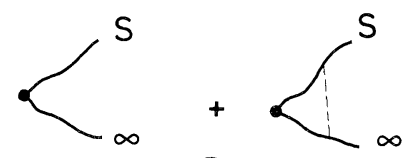

(a)
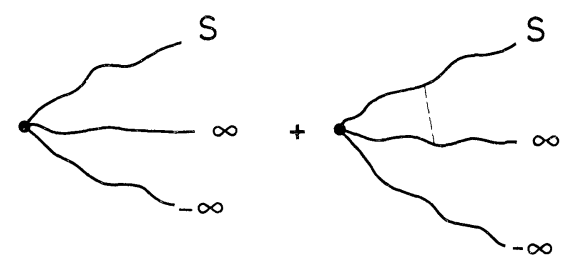

(b)
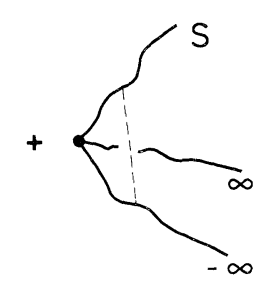

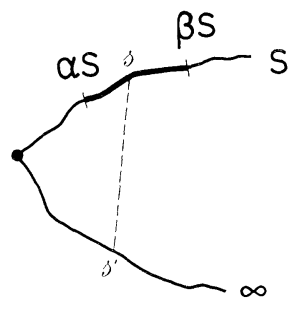

(c)

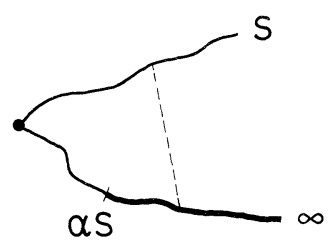

(d)

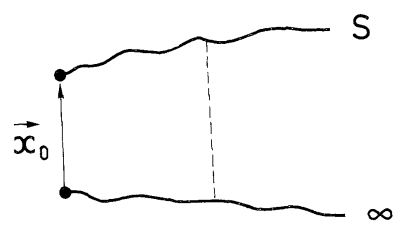

(e)

Fig. 5a-e. The first order diagrams contributing in the continuum theory (2.1) to the probabilities depicted in Fig. 4. The discrete number $n$ of steps of the walks are replaced by the continuum variable $S(2.2)$. The various geometrical cases are labelled in accordance to Fig. 4 , and correspond respectively to probabilities $\mathscr{Z}(S, \infty ; \mathbf{0}, \mathbf{0})(2.34), \mathscr{Z}(S,+\infty,-\infty)(2.36), \mathscr{Z}_{c}(2,38)$, $\mathscr{Z}_{d}(2.40)$ and $\mathscr{Z}_{e}(2.41)$ of the text

Case $b$. Here we have to sum over two interaction diagrams involving a path and separately the two other infinite ones (Fig. 5b). This is just the double of case a (Fig. 5a) [Eq. (2.34)]

$$
\mathscr{Z}(S,-\infty,+\infty)=1+2 z \frac{1}{(1-d / 2)(2-d / 2)}=1-\frac{4}{\varepsilon} z+\ldots .
$$


So, the coefficient of the pole in $1 / \varepsilon$ is doubled, and repeating the calculation of Sect. 2.c yields immediately

in agreement with (2.30).

$$
\begin{aligned}
& \mathscr{Z}(S,-\infty,+\infty) \approx(\ln S)^{-1}, \quad d=4, \\
& \approx S^{-\varepsilon / 2+\ldots,} \quad d=4-\varepsilon,
\end{aligned}
$$

Case c. The continuum analogue of probability of non-empty intersection (c) is obtained in a slightly different way. First we note that its complement 1-Prob $(c)$ of empty intersection has a continuum expression

$$
\mathscr{Z}=\frac{\int d\left\{\mathbf{r}_{1}\right\} d\left\{\mathbf{r}_{2}\right\} P\left\{\mathbf{r}_{1}, \mathbf{r}_{2}\right\} \delta^{d}\left[\mathbf{r}_{1}(0)\right] \delta^{d}\left[\mathbf{r}_{2}(0)\right]}{\int d\left\{\mathbf{r}_{1}\right\} d\left\{\mathbf{r}_{2}\right\} P_{0}\left\{\mathbf{r}_{1}\right\} P_{0}\left\{\mathbf{r}_{2}\right\} \delta^{d}\left[\mathbf{r}_{1}(0)\right] \delta^{d}\left[\mathbf{r}_{2}(0)\right]} .
$$

It is calculated with the truncated weight

$$
\begin{aligned}
\mathscr{P}\left\{\mathbf{r}_{1}, \mathbf{r}_{2}\right\}= & \exp \left\{-\frac{1}{2} \int_{0}^{S} \mathbf{r}_{1}^{2}(s) d s-\frac{1}{2} \int_{0}^{\infty} \dot{\mathbf{r}}_{2}^{2}\left(s^{\prime}\right) d s^{\prime}\right. \\
& \left.-b \int_{\alpha S}^{\beta S} d s \int_{0}^{\infty} d s^{\prime} \delta^{d}\left[\mathbf{r}_{1}(s)-\mathbf{r}_{2}\left(s^{\prime}\right)\right]\right\},
\end{aligned}
$$

which is to be evaluated in the universal limit $z \rightarrow \infty$. To first order in $b$, we thus have

$$
1-\operatorname{Prob}(c) \rightarrow \mathscr{Z}=1-b\left\langle\int_{\alpha S}^{\beta S} d s \int_{0}^{\infty} d s^{\prime} \delta^{d}\left[\mathbf{r}_{1}(s)-\mathbf{r}_{2}\left(s^{\prime}\right)\right] \delta^{d}\left[\mathbf{r}_{1}(0)\right] \delta^{d}\left[\mathbf{r}_{2}(0)\right]\right\rangle_{0}+\ldots
$$

Hence Prob $(c)$ will correspond (at this order) to the continuum intersection local time

It is calculated at

$$
\mathscr{Z}_{c}=b\left\langle\int_{\alpha S}^{\beta S} d s \int_{0}^{\infty} d s^{\prime} \delta \ldots\right\rangle_{0}+\ldots .
$$

$$
\begin{aligned}
\mathscr{Z}_{c} & =b(2 \pi)^{-d / 2} \int_{\alpha S}^{\beta S} d s \int_{0}^{\infty} d s^{\prime}\left(s+s^{\prime}\right)^{-d / 2} \\
& =-z \frac{1}{(1-d / 2)(2-d / 2)}\left[\beta^{2-d / 2}-\alpha^{2-d / 2}\right] .
\end{aligned}
$$

We see that $\mathscr{Z}_{c}$ does not diverge anymore when $d \rightarrow 4$. One order of divergence has indeed been suppressed by letting the first interaction point to be at an abscissa $\alpha S \neq 0$ from the origin (Fig. 5c). If $\alpha=0$, then (2.38) diverges at $d=4$, recovering case $a$. Now, renormalization theory tells us what to do at this (finite) order. (This of course would work to all orders, when divergences occur.) We substitute $g$ to $z$ and we take the $d=4$ limit of the coefficient of $z$. Hence at first order

$$
\mathscr{Z}_{c}=g \ln (\beta / \alpha)+\ldots .
$$

In four dimensions, we obtain from (2.23)

$$
\mathscr{Z}_{c}=\frac{1}{2 \ln S} \ln (\beta / \alpha)+\ldots
$$


which is just Lawler's result (2.31). It is interesting to note that there is a deep similarity with his derivation in $d=4$ (see in particular the second of [12]). Let us denote $D_{n}=\left\{\Pi_{1}[\alpha n, \beta n] \cap \Pi_{2}(0, \infty) \neq \emptyset\right\}$ and $P\left(D_{n}\right)$ its probability. Then [12] $P\left(D_{n}\right) E\left(I_{n} \mid I_{n} \geqq 1\right)=E\left(I_{n}\right)$, where $E$ is the expectation value of the random variable $I_{n}$ measuring the number of intersections of the two walks. Lawler proves that $E\left(I_{n} \mid I_{n} \geqq 1\right)$ just looks like twice the number of intersections of two walks starting at the same point. Hence he finds

and

$$
E\left(I_{n}\right) \sim C_{6} \ln (\beta / \alpha)
$$

$$
E\left(I_{n} \mid I_{n} \geqq 1\right) \sim 2 C_{6} \ln n
$$

where $C_{6}>0$ is a constant which comes from the Green's function of the random walk. The ratio of the two gives just (2.39a). Hence our numerical integral (2.38) is just the analogue of $E\left(I_{n}\right)$, while $g^{-1}$ corresponds to $E\left(I_{n} \mid I_{n} \geqq 1\right)$.

Case $d$. We have similarly for (2.32) the continuum limit to first order (Fig. 5d)

$$
\mathscr{Z}_{d}=b(2 \pi)^{-d / 2} \int_{0}^{S} d s \int_{\alpha S}^{\infty} d s^{\prime}\left(s+s^{\prime}\right)^{-d / 2}=-z \frac{1}{(1-d / 2)(2-d / 2)}\left[(1+\alpha)^{2-d / 2}-\alpha^{2-d / 2}\right] .
$$

The same strategy $z \rightarrow g$ gives in $d=4$ the renormalized limit

$$
\mathscr{Z}_{d}=g \ln (1+1 / \alpha)+\ldots=\frac{1}{2 \ln S} \ln (1+1 / \alpha)+\ldots
$$

in agreement with $(2.32)$.

Case e. We calculate (2.33) in the continuum theory to first order (Fig. 5e)

$$
\mathscr{Z}_{e}=b\left\langle\int_{0}^{s} d s \int_{0}^{\infty} d s^{\prime} \delta^{d}\left[\mathbf{r}_{1}(s)-\mathbf{r}_{2}\left(s^{\prime}\right)\right] \delta^{d}\left[\mathbf{r}_{1}(0)\right] \delta^{d}\left[\mathbf{r}_{2}(0)-\mathbf{x}_{0}\right]\right\rangle_{0} .
$$

The diagrammatic rules give

$$
\begin{aligned}
\mathscr{Z}_{e} & =b \int \frac{d^{d} q}{(2 \pi)^{d}} \int_{0}^{s} d s \int_{0}^{\infty} d s^{\prime} e^{i \mathbf{q} \cdot \mathbf{x}_{0}} e^{-\frac{1}{2} q^{2}\left(s+s^{\prime}\right)} \\
& =b(2 \pi)^{-d / 2} \int_{0}^{S} d s \int_{0}^{\infty} d s^{\prime} e^{-\frac{x_{0}^{2}}{2\left(s+s^{\prime}\right)}\left(s+s^{\prime}\right)^{-d / 2}} .
\end{aligned}
$$

This can be integrated by parts after taking $\left(s+s^{\prime}\right)$ as a variable, to yield

$$
\mathscr{Z}_{e}=z\left[\alpha^{1-d / 2} \Gamma\left(\frac{d}{2}-1\right)+\int_{0}^{1} d x e^{-\frac{\alpha}{x}}\left(x^{1-d / 2}-x^{-d / 2}\right)\right],
$$

where $\alpha=x_{0}^{2} / 2 S$. This is finite when $d \rightarrow 4$. Here again the dominant behaviour for $S \rightarrow \infty$, and $d=4$, is obtained by renormalization, which here amounts to substitute 
$g$ to $z$ and let $d \rightarrow 4$ :

$$
\begin{aligned}
\mathscr{Z}_{e} & =g\left[\frac{1}{\alpha}\left(1-e^{-\alpha}\right)-E_{i}(-\alpha)\right]+\ldots \\
& =\frac{1}{2 \ln S}\left[\frac{1}{\alpha}\left(1-e^{-\alpha}\right)-E_{i}(-\alpha)\right]+\ldots
\end{aligned}
$$

This corrects Lawler's statement (2.33). [Note that the coefficient of $(\ln S)^{-1}$ must precisely be universal and not lattice dependent.]

\section{Intersections of Three Walks Near Three Dimensions}

This case has been considered by Lawler in [13] (see also [11]). Let $S_{1}(n, \omega), S_{2}(n, \omega)$, $S_{3}(n, \omega)$ be independent simple random walks in $\mathbb{Z}^{3}$, with transition probabilities [13]

$$
P\left\{S_{i}(n+1)-S_{i}(n)=e \mid S_{i}(n)\right\}=\frac{1}{6}, \quad i=1,2,3, \quad|e|=1,
$$

$e$ being a lattice unit vector in $\mathbb{Z}^{3}$.

As above, the trajectories are

$$
\begin{aligned}
& \Pi_{i}[a, b]=\left\{S_{i}(j, \omega): a<j<b\right\}, \\
& \Pi_{i}(a, b)=\left\{S_{i}(j, \omega): a \leqq j \leqq b\right\},
\end{aligned}
$$

etc.... One assumes one of the walks $(i=1)$ to start at the origin, and the two other ones to start at $\mathbf{x}_{0}$. Lawler studied [13] the probability that

$$
\Pi_{1}\left[a_{1}, b_{1}\right] \cap \Pi_{2}\left[a_{2}, b_{2}\right] \cap \Pi_{2}\left[a_{3}, b_{3}\right] \neq \emptyset
$$

when $\mathbf{x}_{0} \neq \mathbf{0}$, and for $\mathbf{x}_{0}=\mathbf{0}$ the probability that

$$
\Pi_{1}\left(a_{1}, b_{1}\right] \cap \Pi_{2}\left[a_{2}, b_{2}\right] \cap \Pi_{2}\left[a_{3}, b_{3}\right]=\emptyset .
$$

In [11] it was shown that, with probability one,

$$
\Pi_{1}[0, \infty) \cap \Pi_{2}[0, \infty) \cap \Pi_{3}[0, \infty) \neq \varnothing
$$

regardless of what $\mathbf{x}_{0}$ is. Hence, three infinite walks always intersect in $\mathbb{Z}^{3}$. So one is interested in the case where at least one of the paths is finite. Now, if one considers instead three independent continuous Wiener processes $X_{1}, X_{2}, X_{3}$ in $\mathbb{R}^{3}$, and let $\Gamma_{i}(a, b)$ denote the random trajectories $\Gamma_{i}(a, b)=\left\{X_{i}(t): a \leqq t \leqq b\right\}$, then with probability one

$$
\Gamma_{1}(0, \infty) \cap \Gamma_{2}(0, \infty) \cap \Gamma_{3}(0, \infty)=\emptyset
$$

when the processes start at different points. Hence $d=3$ is a critical dimension for intersections of three walks or paths, like $d=4$ was for two walks. This is in relation to a well-known result in the theory of critical phenomena [27]:d=3 is the upper critical dimension for tricritical phenomena, which are modelized by a $\left(\varphi^{2}\right)_{d}^{3}$ interaction term in field theory [27]. This $\left(\varphi^{2}\right)_{d}^{3}$ interaction term has precisely a Brownian path representation where three paths intersect [21,25]. This tricritical field theoretic representation has been originally used [21, 39-42] for studying 
polymers near the so-called $\Theta$-point. At this point, excluded volume, i.e. two-body interactions, vanish, and one is lead to consider the next-order effect of three-body interactions of chains with themselves or with other chains. The $\Theta$-point is so identified as a tricritical point [21]. The direct analytic theory of such three-body interactions is now well established $[41,42]$ for polymer chains. We shall use here a new variant of it, in order to describe the mutual intersection properties of three random walks, in a space dimension $d \leqq 3$. Let us first recall some expected results.

\section{3.a. Lawler's Results}

We briefly sketch the essential results of [13].

For three walks all starting at the origin in $\mathbb{Z}^{3}$ :

(a) There exist positive constants $k_{1}, k_{2}$ such that

$$
k_{1}(\ln n)^{-1} \leqq f(n) \equiv P\left\{\Pi_{1}(0, n] \cap \Pi_{2}[0, \infty) \cap \Pi_{3}[0, \infty)=\emptyset\right\} \leqq k_{2}(\ln n)^{-1 / 4} \text {. }
$$

An incomplete argument was also given that for any $r>1 / 4$,

$$
\lim (\ln n)^{r} f(n)=+\infty \text {. }
$$

Hence $(\ln n)^{-1 / 4}$ should be the actual decay rate of the probability $f(n)$ of no triple intersections between three walks.

(b) Also, for one-sided walk and two-sided walks, all starting at $\mathbf{0}$,

$$
g(n) \equiv P\left\{\Pi_{1}(0, n] \cap \Pi_{2}(-\infty,+\infty) \cap \Pi_{3}(-\infty,+\infty)=\emptyset\right\} \approx(\ln n)^{-1} .
$$

For the probabilities of non-empty intersections, the results are the following. For $0<\alpha<\beta<\infty$,

(c) $\lim _{n \rightarrow \infty}(\ln n) P\left\{\Pi_{1}(\alpha n, \beta n) \cap \Pi_{2}[0, \infty) \cap \Pi_{3}[0, \infty) \neq \emptyset\right\}=\frac{1}{4} \ln (\beta / \alpha)$.

A constant $c_{12}$ also exists such that

(d) $P\left\{\Pi_{1}[\alpha n, \infty) \cap \Pi_{2}[0,(\beta-\alpha) n] \cap \Pi_{3}[0,(\beta-\alpha) n] \neq \emptyset\right\} \leqq c_{12} \ln (\beta / \alpha)(\ln n)^{-1}$,

(e) $P\left\{\Pi_{1}[0,(\beta-\alpha) n] \cap \Pi_{2}[\alpha n, \infty) \cap \Pi_{3}[\alpha n, \infty) \neq \emptyset\right\} \leqq c_{12} \ln (\beta / \alpha)(\ln n)^{-1}$.

Instead of these upperbounds (3.4) (3.5), we shall obtain the exact asymptotic forms $\mathscr{I}(\beta / \alpha)(\ln n)^{-1}, \mathscr{J}(\beta / \alpha)(\ln n)^{-1}$, where $\mathscr{I}$ and $\mathscr{J}$ will be calculated explicitely. Finally, for three walks $S_{1}, S_{2}, S_{3}$ starting at $\mathbf{x}_{0}, \mathbf{0}, \mathbf{0}$ respectively, one has [13]

$$
P\left\{\Pi_{1}[0, n] \cap \Pi_{2}[0, \infty) \cap \Pi_{3}[0, \infty) \neq \emptyset\right\} \approx(\ln n)^{-1} .
$$

We shall here derive (3.7) together with its finite universal coefficient.

We shall evaluate (3.1)-(3.7), by using a direct renormalization approach, which is the analogue of that of Sect. 2 for two walks. It is an extension of the tricritical theory that we devised for polymers in [40-42]. Here the walks are not any more self-avoiding but only mutually avoiding. As we shall see, the technique is extremely simple. 


\section{3.b. The Three-Body Continuum Model and Its Renormalization}

The probability weight describing three-point intersections is a generalization of Edwards' model (1.9) in $\mathbb{R}^{d}$,

$$
\begin{aligned}
\mathscr{P}\left\{\mathbf{r}_{1}, \mathbf{r}_{2}, \mathbf{r}_{3}\right\}= & \exp \left\{-\frac{1}{2} \int_{0}^{s_{1}} d s_{1} \dot{\mathbf{r}}_{1}^{2}\left(s_{1}\right)-\frac{1}{2} \int_{0}^{s_{2}} d s_{2} \dot{\mathbf{r}}_{2}^{2}\left(s_{2}\right)-\frac{1}{2} \int_{0}^{s_{3}} d s_{3} \dot{\mathbf{r}}_{3}^{2}\left(s_{3}\right)\right. \\
& \left.-c \int_{0}^{s_{1}} d s_{1} \int_{0}^{s_{2}} d s_{2} \int_{0}^{s_{3}} d s_{3} \delta^{d}\left[\mathbf{r}_{1}\left(s_{1}\right)-\mathbf{r}_{2}\left(s_{2}\right)\right] \delta^{d}\left[\mathbf{r}_{2}\left(s_{2}\right)-\mathbf{r}_{3}\left(s_{3}\right)\right]\right\} .
\end{aligned}
$$

This weight, and the double local time involved in it $\int d s_{1} d s_{2} d s_{3} \delta^{d} \delta^{d}$, have not yet been studied rigorously, and furnish an interesting problem. However, from a practical point of view, their analytical manipulation is fairly well-defined [40-42], and efficient. As for Edwards' model (1.9), we work in continuum dimension $d$, with dimensional regularization [42]. This means that all quantities are defined by analytical continuation from $d<2[38,42]$. More precisely, since $d=3$ is the upper tricritical dimension, we shall set

$$
d=3-\varepsilon^{\prime},
$$

and after renormalization, we shall reach the limit $\varepsilon^{\prime} \rightarrow 0$. The dimensionless expansion parameter associated with the three-body interaction term in (3.8) is defined as $[40,41]$

$$
y=(2 \pi)^{-d} c S^{3-d},
$$

where $S$ is one of the three "areas" $S_{1}, S_{2}, S_{3}$, and is the finite one if some of these are infinite. The method is then the following: calculate partition functions in perturbation theory of $y$, for $d<3$, then renormalize poles in $1 / \varepsilon^{\prime}$, by substituting in scaling functions a three-body renormalized coupling constant [40] $h$ to $y$, to be defined below. Calculate via the Wilson function, the fixed point value $h^{*}\left(\varepsilon^{\prime}\right)$ of $h$ for $d=3-\varepsilon^{\prime}$, or its logarithmic asymptotic form for $d=3$. This program is entirely analogous to that followed in Sect. 2 for $z, g, W[g, \varepsilon]$ in the case of two-body interactions in a space dimension $d=4-\varepsilon$. We shall need the dimensionless "thirdvirial coefficient" $h$, defined by analogy to (2.16) (2.17),

$$
h=\mathscr{Z}_{3}(S, S, S)(2 \pi S)^{-d},
$$

where $\mathscr{Z}_{3}(S, S, S)$ is the connected partition function of three identical walks of areas $S$ weighted by (3.8)

$$
\mathscr{Z}_{3}(S, S, S)=c\left\langle\int_{0}^{S} d s_{1} \int_{0}^{S} d s_{2} \int_{0}^{S} d s_{3} \delta^{d}\left[\mathbf{r}_{1}\left(s_{1}\right)-\mathbf{r}_{2}\left(s_{2}\right)\right] \delta^{d}\left[\mathbf{r}_{2}\left(s_{2}\right)-\mathbf{r}_{3}\left(s_{3}\right)\right] \delta^{d}\left[\mathbf{r}_{1}(0)\right]\right\rangle .
$$

This is immediately interpreted as the local time measure of the random configurations where the trajectories of three walks, starting anywhere (with however one of the origins fixed for eliminating a trivial volume factor), have at least one point in common. 

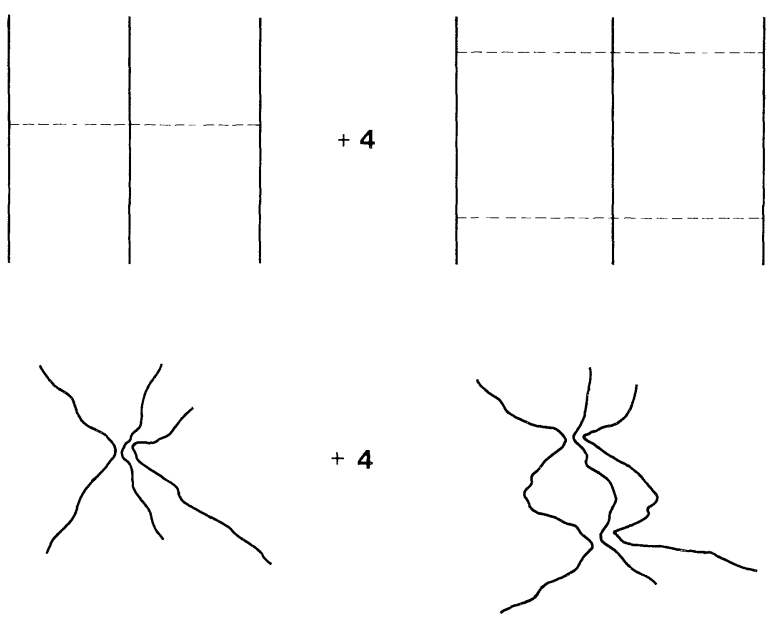

Fig. 6. First order diagrams and configurations contributing to the connected partition function $\mathscr{Z}_{3}(S, S, S)(3.12)$ of three Brownian paths having a triple point in common

The first order diagrams necessary to calculate $\mathscr{Z}_{3}$ are shown in Fig. 6. The rules are those given in Sect. 2. We have after a Gaussian integration over the momenta,

$$
\mathscr{Z}_{3}=c S^{3}-4 c^{2}(2 \pi)^{-d} \int_{0}^{S} d s \int_{0}^{S} d s^{\prime} \int_{0}^{S} d s^{\prime \prime}(S-s)\left(S-s^{\prime}\right)\left(S-s^{\prime \prime}\right)\left(s s^{\prime}+s^{\prime} s^{\prime \prime}+s^{\prime \prime} s\right)^{-d / 2}
$$

The dominant diverging part for $d=3-\varepsilon^{\prime}$ is easily evaluated to be

$$
\mathscr{Z}_{3}=c S^{3}\left(1-y \frac{8 \pi}{\varepsilon^{\prime}}\right) \text {. }
$$

Hence the dimensionless "third virial coefficient" $h(3.11)$ reads in terms of the bare parameter $y(3.10)$,

$$
h=y\left(1-\frac{8 \pi}{\varepsilon^{\prime}} y+\ldots\right)
$$

The Wilson function of $h$ is defined as in Eq. (2.20),

$$
W\left(y, \varepsilon^{\prime}\right)=\left.W\left[h, \varepsilon^{\prime}\right] \equiv S \frac{\partial h}{\partial S}\right|_{c, \varepsilon^{\prime}}=\left.\varepsilon^{\prime} y \frac{\partial}{\partial y} h\right|_{\varepsilon^{\prime}} .
$$

Using Eq. (3.15) yields immediately

$$
W\left(y, \varepsilon^{\prime}\right)=\varepsilon^{\prime} y-16 \pi y^{2}+\ldots,
$$

and by substituting $h$ to $y$

$$
W\left[h, \varepsilon^{\prime}\right]=\varepsilon^{\prime} h-8 \pi h^{2}+\ldots .
$$

This double expansion in $h$ and $\varepsilon^{\prime}$ is regular to all orders in $h$, and this non-trivial result of renormalization theory could be derived by the same method as in [41] for 
the standard three-body interaction of polymers. The fixed point value of $h$ for $d=3-\varepsilon^{\prime}$ satisfies $W\left[h^{*}, \varepsilon^{\prime}\right]=0$ and reads

$$
h^{*}\left(\varepsilon^{\prime}\right)=\frac{\varepsilon^{\prime}}{8 \pi}+O\left(\varepsilon^{\prime 2}\right)
$$

For $d=3$ instead, we first consider the finite limit of the Wilson function

$$
W[h, 0]=\left.S \frac{\partial}{\partial S} h\right|_{c, \varepsilon^{\prime}=0}=-8 \pi h^{2}+O\left(h^{3}\right) .
$$

This is immediately integrated into

$$
h=1 /(8 \pi \ln S)+O\left(\ln \ln S / \ln ^{2} S\right)
$$

This asymptotic expression of $h$ is universal [27,40], and will dominate all the intersection statistics of three random walks in three dimensions, as we shall see now.

\section{3.c. Intersections of Three Walks}

(a) Let us first consider the probability $f(n)$ (3.1) that three walks all starting at $\mathbf{0}$ do not intersect again (Fig. 7a). The associated continuum partition function is simply

$$
\begin{aligned}
& \mathscr{Z}_{a}\left(S_{1}, S_{2}, S_{3}\right) \\
& \quad=\frac{\int d\left\{\mathbf{r}_{1}\right\} d\left\{\mathbf{r}_{2}\right\} d\left\{\mathbf{r}_{3}\right\} \mathscr{P}\left\{\mathbf{r}_{1}, \mathbf{r}_{2}, \mathbf{r}_{3}\right\} \delta^{d}\left[\mathbf{r}_{1}(0)\right] \delta^{d}\left[\mathbf{r}_{2}(0)\right] \delta^{d}\left[\mathbf{r}_{3}(0)\right]}{\int d\left\{\mathbf{r}_{1}\right\} d\left\{\mathbf{r}_{2}\right\} d\left\{\mathbf{r}_{3}\right\} \mathscr{P}_{0}\left\{\mathbf{r}_{1}\right\} \mathscr{P}_{0}\left\{\mathbf{r}_{2}\right\} \mathscr{P}_{0}\left\{\mathbf{r}_{3}\right\} \delta^{d}\left[\mathbf{r}_{1}(0)\right] \delta^{d}\left[\mathbf{r}_{2}(0)\right] \delta^{d}\left[\mathbf{r}_{3}(0)\right]},
\end{aligned}
$$

where $\mathscr{P}$ is weight (3.8). The first order contribution to $\mathscr{Z}_{a}$ in powers of $c$ or $y$ is given in Fig. 7a:

$$
\mathscr{Z}_{a}=1-c(2 \pi)^{-d} \int_{0}^{S_{1}} d s \int_{0}^{S_{2}} d s^{\prime} \int_{0}^{S_{3}} d s^{\prime \prime}\left(s s^{\prime}+s^{\prime} s^{\prime \prime}+s^{\prime \prime} s\right)^{-d / 2}
$$

This integral diverges, as expected, for $d=3$, when $s, s^{\prime}, s^{\prime \prime}$ go to zero simultaneously. Evaluating the residue of the pole in $1 / \varepsilon^{\prime}$ is easily performed. For instance one sets $s=u^{-1}, \ldots$, and uses the integral representation

$$
\left(u+u^{\prime}+u^{\prime \prime}\right)^{-d / 2}=\int_{0}^{\infty} d p e^{-p\left(u+u^{\prime}+u^{\prime \prime}\right)} p^{d / 2-1} / \Gamma(d / 2) .
$$

We find in the various limits of $S_{1}, S_{2}, S_{3}$,

and

$$
\mathscr{Z}_{a}(S, S, S)=1-y\left(\frac{2 \pi}{\varepsilon^{\prime}}+\ldots\right)+\ldots
$$

$$
\mathscr{Z}_{a}(S, \infty, \infty)=1-y\left(\frac{2 \pi}{\varepsilon^{\prime}}+\ldots\right)+\ldots
$$




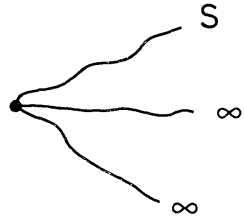

(a)
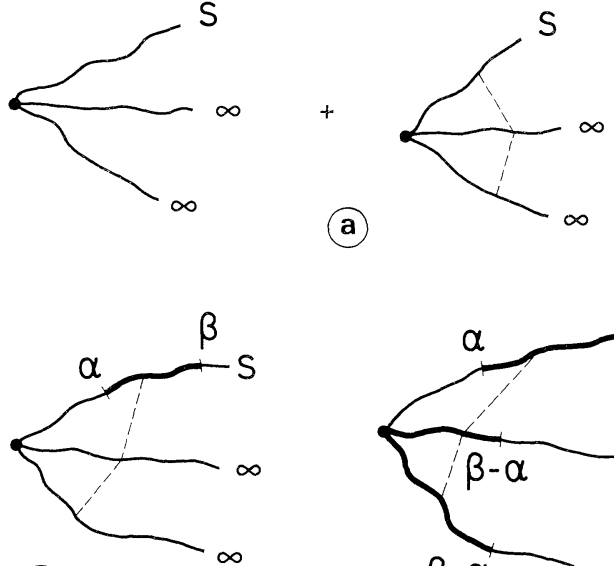

(C)

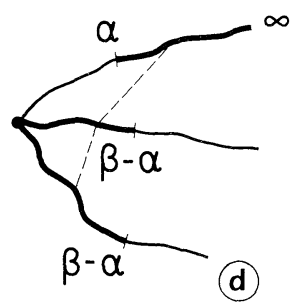

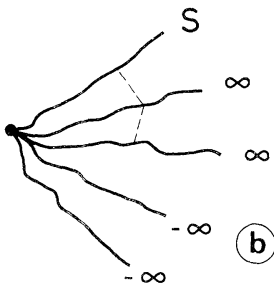
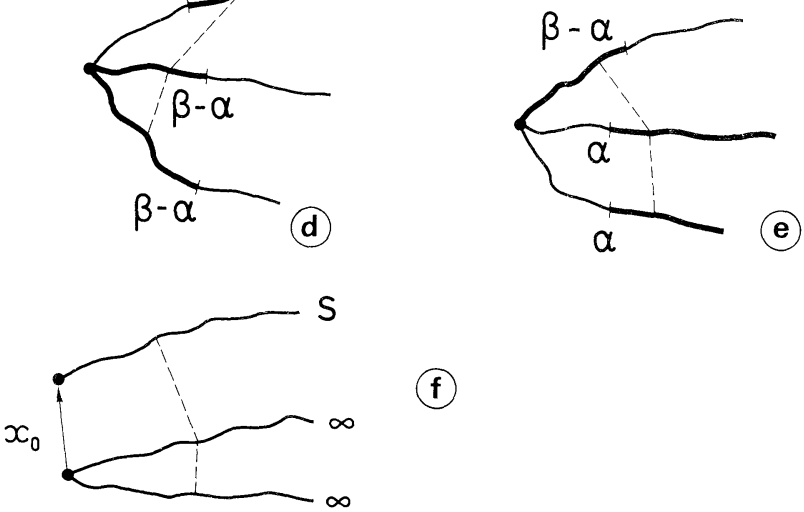

(f)

Fig. 7a-f. Diagrams contributing to probabilities $\mathscr{Z}_{i}, i=\mathbf{a}, \mathbf{b}, \mathbf{c}, \mathbf{d}, \mathbf{e}, \mathbf{f}$ of Sect. 3 c. The interactions are only those of triple points occurring in the continuum model (3.8)

only subdominant terms being modified by the limit $S_{2}, S_{3} \rightarrow \infty$. The scaling behaviour of $\mathscr{Z}_{a} \sim S^{\zeta}$ for $d<3$ is then obtained by introducing a Wilson function:

$$
\zeta\left(y, \varepsilon^{\prime}\right)=\zeta\left[h, \varepsilon^{\prime}\right]=\left.S \frac{\partial}{\partial S} \ln \mathscr{Z}_{a}\right|_{c, \varepsilon^{\prime}}=-2 \pi y+\ldots=-2 \pi h+O\left(h^{2}\right) .
$$

In terms of $h$ and $\varepsilon^{\prime}$ the important result of renormalization theory is of course that $\zeta\left[h, \varepsilon^{\prime}\right]$ is regular to all orders in $h$ when $\varepsilon^{\prime} \rightarrow 0$. So in $d=3-\varepsilon^{\prime}$, we find from result (3.18) the fixed point value of $\zeta$

$$
\zeta\left[h^{*}, \varepsilon^{\prime}\right]=-\frac{\varepsilon^{\prime}}{4}+O\left(\varepsilon^{\prime 2}\right)
$$
In exactly $d=3,(3.23)$ (3.19) give together $S \frac{\partial}{\partial S} \ln \mathscr{Z}_{a}=-\frac{1}{4 \ln S}+\ldots$, which is
integrated into

$$
\mathscr{Z}_{a}=\text { const }(\ln S)^{-1 / 4} .
$$

This fully agrees with Lawler's probabilistic statements (3.1)(3.2), which is thus valid both for $\mathscr{Z}_{a}(S, S, S)$ and $\mathscr{Z}_{a}(S, \infty, \infty)$.

(b) The two walks $S_{2}, S_{3}$ are now infinite and two-sided (Eq. 3.3). Then the partition function $\mathscr{Z}_{b}$ which is the continuum analogue of probability $g(n)(3.3)$, is defined in a obvious way as in (3.20). Its first order expansion (Fig. 7b) is simply 
obtained from (3.22),

$$
\mathscr{Z}_{b}=1-4 \times \frac{2 \pi}{\varepsilon^{\prime}} y,
$$

the extra factor 4 coming simply from the 4 analogous diagrams in Fig. $7 \mathrm{~b}$, to be compared to Fig. 7a. This gives immediately in $d=3-\varepsilon^{\prime}$ from (3.24)

and in $d=3$ from $(3.25)$

$$
\mathscr{Z}_{b} \approx \mathrm{S}^{-\varepsilon^{\prime}+0\left(\varepsilon^{\prime 2}\right)}
$$

$$
\mathscr{Z}_{b} \approx(\ln S)^{-1} \text {. }
$$

This agrees with probabilistic estimation (3.3) for $g(n)$.

(c) Probability of intersection of a truncated walk $\Pi_{1}(\alpha n, \beta n)$, with two infinite ones $\Pi_{2}[0, \infty)$ and $\Pi_{3}[0, \infty)$. For evaluating (3.4), which is a probability of nonempty intersection, we use the same technique as in Sect. 2, case $c$. In particular, there is no critical divergence in first order in $y$, and its suffices to renormalize the coupling constant $y$ into $h$. More precisely, the probability

$$
P\left\{\Pi_{1}(\alpha n, \beta n) \cap \Pi_{2}[0, \infty) \cap \Pi_{3}[0, \infty) \neq \emptyset\right\}
$$

has a continuum expression to first order in $c$

$$
\begin{aligned}
\mathscr{Z}_{c}= & c\left\langle\int_{\alpha S}^{\beta S} d s \int_{0}^{\infty} d s^{\prime} \int_{0}^{\infty} d s^{\prime \prime} \delta^{d}\left[\mathbf{r}_{1}(s)-\mathbf{r}_{2}\left(s^{\prime}\right)\right] \delta^{d}\left[\mathbf{r}_{2}\left(s^{\prime}\right)-\mathbf{r}_{3}\left(s^{\prime \prime}\right)\right]\right. \\
& \left.\cdot \delta^{d}\left[\mathbf{r}_{1}(0)\right] \delta^{d}\left[\mathbf{r}_{2}(0)\right] \delta^{d}\left[\mathbf{r}_{3}(0)\right]\right\rangle_{0}+\ldots
\end{aligned}
$$

where the average is evaluated at this order with the pure Brownian weight $(c=0)$. The evaluation of (3.28) is done with the help of diagram $c$ of Fig. 7,

$$
\mathscr{Z}_{c}=c(2 \pi)^{-d} \int_{\alpha S}^{\beta S} d s \int_{0}^{\infty} d s^{\prime} \int_{0}^{\infty} d s^{\prime \prime}\left(s s^{\prime}+s^{\prime} s^{\prime \prime}+s^{\prime \prime} s\right)^{-d / 2} .
$$

The multiple integral converges for $d=3$. We change the variables: $s=S u^{-2}$ and get

$$
\mathscr{Z}_{c}=y 8 \int_{\beta^{-1 / 2}}^{\alpha^{-1 / 2}} d u \int_{0}^{\infty} d u^{\prime} \int_{0}^{\infty} d u^{\prime \prime}\left(u u^{\prime} u^{\prime \prime}\right)^{d-3}\left(u^{2}+u^{\prime 2}+u^{\prime \prime 2}\right)^{-d / 2} .
$$

The integral simplifies in $d=3$, and is evaluated to be

$$
\mathscr{Z}_{\mathrm{c}}=y 2 \pi \ln (\beta / \alpha) .
$$

Obviously, this is only a first order calculation in $y$. But renormalization theory again tells us that the asymptotic result for $S$ large is simply obtained by substituting $h$ to $y$, which could be checked by calculating next order terms. Hence the true asymptotic behaviour of $\mathscr{Z}_{c}$ is

$$
\mathscr{Z}_{c}=h 2 \pi \ln (\beta / \alpha)+\ldots=\frac{1}{4} \ln (\beta / \alpha) / \ln S+\ldots,
$$

where we used (3.19). This is just Lawler's result (3.4).

$$
P\left\{\Pi_{1}[\alpha n, \infty) \cap \Pi_{2}[0,(\beta-\alpha) n] \cap \Pi_{3}[0,(\beta-\alpha) n] \neq \emptyset\right\} .
$$


The continuum equivalent of this probability is, to first order, simply a variant of (3.29) (Fig. 7d)

$$
\mathscr{Z}_{d}=c(2 \pi)^{-d} \int_{\alpha S}^{\infty} d s \int_{0}^{(\beta-\alpha) S} d s^{\prime} \int_{0}^{(\beta-\alpha) S} d s^{\prime \prime}\left(s s^{\prime}+s^{\prime} s^{\prime \prime}+s^{\prime \prime} s\right)^{-d / 2}+\ldots .
$$

Integration over $s$ yields in $d=3$,

with

$$
\mathscr{Z}_{d}=2 y \mathscr{J}(\beta / \alpha)
$$

$$
\mathscr{I}(\beta / \alpha)=\int_{0}^{\frac{\beta}{\alpha}-1} d x d y(x+y)^{-1}(x+y+x y)^{-1 / 2} .
$$

Now, in the large scale limit $S \rightarrow \infty$, the resummation of the perturbation series in $y$ amounts to a simple substitution $y \rightarrow h(3.19)$ and finally:

$$
\mathscr{Z}_{d}=\mathscr{I}(\beta / \alpha) /(4 \pi \ln S)+\ldots .
$$

Note that the function $\mathscr{I}(\beta / \alpha)$ is not of the form $\ln (\beta / \alpha)$ appearing in (3.5). Moreover $\mathscr{I}$ can be evaluated for $\beta / \alpha-1 \ll 1$,

$$
\mathscr{I}(\beta / \alpha) \approx \iint_{0}^{\frac{\beta}{\alpha}-1} d x d x(x+y)^{-3 / 2}=4(2-\sqrt{2})(\beta / \alpha-1)^{1 / 2},
$$

which grows faster than $\ln (\beta / \alpha) \approx \beta / \alpha-1$. Hence our result (3.34) (3.35), contradicts Lawler's explicit upper bound (3.5), for which we think that the coefficient of $(\ln n)^{-1}$ should be corrected into (3.33).

$$
P\left\{\Pi_{1}[0,(\beta-\alpha) n] \cap \Pi_{2}[\alpha n, \infty) \cap \Pi_{3}[\alpha n, \infty) \neq \emptyset\right\} .
$$

This probability corresponds to the physical situation depicted in Fig. 7e, and its continuum analogue reads to first order in $c$ :

$$
\mathscr{Z}_{e}=c(2 \pi)^{-d} S^{3-d} \int_{0}^{\beta-\alpha} d s \int_{\alpha}^{\infty} d s^{\prime} \int_{\alpha}^{\infty} d s^{\prime \prime}\left(s s^{\prime}+s^{\prime} s^{\prime \prime}+s^{\prime \prime} s\right)^{-d / 2} .
$$

Again this integral converges $d=3$. Integrating on $s$ and renormalizing $y$ into $h$ (3.19), we find the universal asymptotic result

$$
\mathscr{Z}_{e}=\frac{1}{4 \pi \ln S} \mathscr{J}(\beta / \alpha)+\ldots
$$

with

$$
\mathscr{J}(\beta / \alpha) \equiv \int_{\left(\frac{\beta}{\alpha}-1\right)^{-1}}^{\infty} d x d y \frac{1}{x+y}\left[\frac{1}{(x y)^{1 / 2}}-\frac{1}{(x+y+x y)^{1 / 2}}\right]
$$

This provides the expected asymptotic form, ameliorating the upper bound (3.6). Both for $\beta \rightarrow \alpha$, and $\beta \rightarrow \infty$, (3.37) (3.38) do agree with (3.6).

(f) Let us finally consider the probability

$$
P\left\{\Pi_{1}[0, n] \cap \Pi_{2}[0, \infty) \cap \Pi_{3}[0, \infty) \neq \emptyset\right\}
$$


that one walk starting at $\mathbf{x}_{0}$, and two others starting at $\mathbf{0}$, have a non-empty three point intersection in $\mathbb{Z}^{3}$ (Fig. 7f). The continuum analogue of this probability is

$$
\operatorname{Prob}(f) \rightarrow 1-\mathscr{Z}_{f}^{\prime},
$$

where $\mathscr{Z}_{f}^{\prime}$ is the partition function

$$
\mathscr{Z}_{f}^{\prime}=\left\langle\delta^{d}\left[\mathbf{r}_{1}(0)-\mathbf{x}_{0}\right] \delta^{d}\left[\mathbf{r}_{2}(0)\right] \delta^{d}\left[\mathbf{r}_{3}(0)\right]\right\rangle
$$

calculated with the weight (3.8), in the limit $y=c(2 \pi)^{-d} S^{3-d} \rightarrow \infty$. To zeroth order $\mathscr{Z}_{f}^{\prime}=1$. Hence Prob $(f)$ reads to first order in $c$ :

$$
\begin{aligned}
\operatorname{Prob}(f) \rightarrow \mathscr{Z}_{f} \equiv & c\left\langle\int_{0}^{s} d s \int_{0}^{\infty} d s^{\prime} \int_{0}^{\infty} d s^{\prime \prime} \delta^{d}\left[\mathbf{r}_{1}(s)-\mathbf{r}_{2}\left(s^{\prime}\right)\right] \delta^{d}\left[\mathbf{r}_{2}\left(s^{\prime}\right)-\mathbf{r}_{3}\left(s^{\prime \prime}\right)\right]\right. \\
& \left.\cdot \delta^{d}\left[\mathbf{r}_{1}(0)-\mathbf{x}_{0}\right] \delta^{d}\left[\mathbf{r}_{2}(0)\right] \delta^{d}\left[\mathbf{r}_{3}(0)\right]\right\rangle_{0}+\ldots,
\end{aligned}
$$

which corresponds to the interaction diagram $f$ of Fig. 7. The Brownian average (3.40) is evaluated as usual

$$
\begin{gathered}
\mathscr{Z}_{f}=y \mathscr{I}_{d}(\alpha) \\
\mathscr{I}_{d}(\alpha) \equiv \int_{0}^{1} d s \int_{0}^{\infty} d s^{\prime} d s^{\prime \prime}\left(s s^{\prime}+s^{\prime} s^{\prime \prime}+s^{\prime \prime} s\right)^{-d / 2} e^{-\alpha\left(s^{\prime}+s^{\prime \prime}\right) /\left(s s^{\prime}+s^{\prime} s^{\prime \prime}+s^{\prime \prime} s\right)},
\end{gathered}
$$

where we have rescaled variables by $S$, and set

$$
\alpha \equiv x_{0}^{2} / 2 S \text {, }
$$

Integral (3.42) does converge for $d=3$. Therefore renormalization theory gives the asymptotic form of $\mathscr{Z}_{f}$ as before for $d=3$,

$$
\mathscr{Z}_{f}=h \mathscr{I}_{d=3}(\alpha)+\ldots=\frac{1}{8 \pi \ln S} \mathscr{I}_{d=3}(\alpha)+\ldots .
$$

The integral $\mathscr{I}_{3}(\alpha)(3.42)$ can be evaluated by setting $s=x^{-2}, \ldots$,

$$
\mathscr{I}_{3}(\alpha)=2^{3} \int_{1}^{\infty} d x \int_{0}^{\infty} d y d z\left(x^{2}+y^{2}+z^{2}\right)^{-3 / 2} e^{-\alpha x^{2} \frac{\left(y^{2}+z^{2}\right)}{x^{2}+y^{2}+z^{2}}} .
$$

Integrating over the angle variable in polar coordinates for $y, z$, and performing the change of variables $u=x /\left(x^{2}+y^{2}+z^{2}\right)^{1 / 2}, v=x^{2}\left(1-u^{2}\right)$, one finds

$$
\mathscr{I}_{3}(\alpha)=2 \pi \int_{0}^{1} d u \int_{-u^{2}}^{\infty} e^{-\alpha v} \frac{d v}{v}
$$

which gives finally, after integration by parts on $u$, and with use of $x=1-u^{2}$,

$$
\mathscr{I}_{3}(\alpha)=2 \pi\left\{\int_{0}^{1}[1-\sqrt{1-x}] e^{-\alpha x} \frac{d x}{x}-E_{i}(-\alpha)\right\} \text {. }
$$

This, together with (3.44), yields the universal coefficient of the expected asymptotic behavior (3.7), as announced in the introduction (Eq. (1.8)). 


\section{Higher Order Intersections}

\section{4.a. Model}

We shall now generalize the above results to the case where $P$ walks $(P \geqq 2)$ simultaneously intersect. This case is briefly alluded in ref. [10]. We shall use for this purpose the generalization of probability weights (1.9), (3.8),

$$
\mathscr{P}\left\{\mathbf{r}_{i}\right\}=\exp \left\{-\frac{1}{2} \sum_{i=1}^{P} \int_{0}^{S_{i}} d s_{i} \dot{\mathbf{r}}_{i}^{2}\left(s_{i}\right)-b \prod_{i=1}^{P} \int_{0}^{S_{i}} d s_{i} \prod_{i=1}^{P-1} \delta^{d}\left[\mathbf{r}_{i}\left(s_{i}\right)-\mathbf{r}_{i+1}\left(s_{i+1}\right)\right]\right\},
$$

where the local time of intersection is now a product of $P-1$ Dirac distributions. $b>0$ as usual, plays the role of the repulsive coupling constant. First, dimensional analysis shows that the dimensionless parameter of the $P$-body interaction can be chosen as

$$
z=(2 \pi)^{-\frac{d}{2}(P-1)} b S^{P-\frac{d}{2}(P-1)},
$$

which generalizes (2.3) for $P=2$ and (3.10) for $P=3$.

Hence the upper critical dimension for intersections of $P$ walks is given by

$$
d^{*}=\frac{2 P}{P-1}
$$

and for $P \geqq 4, d^{*}$ becomes less than $d=3$, with an accumulation point at $d=2$ for $P \rightarrow \infty$. Hence this critical dimension does not really correspond to a physical situation. However, the probabilistic nature of the problem does not really require $d$ to be an integer, and the results have certainly a mathematical meaning. Now, for $d>d^{*}$, the interaction parameter $z$ vanishes in the asymptotic limit of long walks $S \rightarrow \infty$. So $P$-intersections will form an empty set for $d>d^{*}$. On the contrary, for

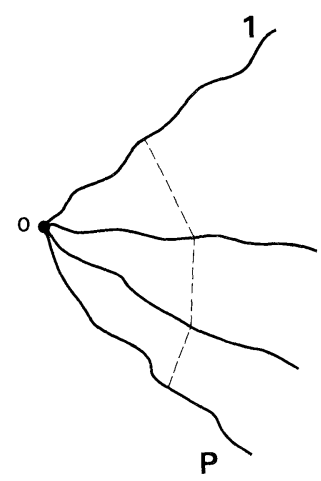

(a)

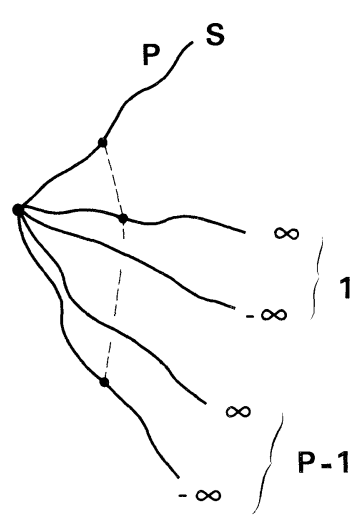

(b)

Fig. 8a and b. Intersections of order $P$ of the trajectories of $P$ Brownian paths. Diagram a contributes to the probability (4.4), or continuum partition function (4.5), that $P$ trajectories do not intersect all together. Diagram b corresponds to the same probability, but for one single path and $P-1$ two-sided paths 
$d<d^{*}, P$-walk intersections are relevant $(z \rightarrow \infty)$, and the theory will reach a fixed point limit [as a $\left(\varphi^{2}\right)_{d}^{P}$ scalar theory]. At the edge $d=d^{*}$, one expects logarithmic corrections for walks on $\mathbb{Z}^{d^{*}}$, with a trivial limit in the continuum space $\mathbb{R}^{d^{*}}$.

Let us consider the probability that $P$ walks, starting at the origin in $\mathbb{Z}^{d}$, have an empty multiple intersection set:

$$
P\left\{\Pi_{1}\left[0, n_{1}\right] \cap \ldots \cap \Pi_{P}\left[0, n_{P}\right]=\emptyset\right\} .
$$

Its continuum analogue, is, like in the above sections, the partition function

$$
\mathscr{Z}=\left\langle\prod_{i=1}^{P} \delta^{d}\left[\mathbf{r}_{i}(0)\right]\right\rangle,
$$

where the average is taken with the weight (4.1), as a functional integral like in Eq. (2.5), and normalized with respect to the pure Brownian motions. Calculating $\mathscr{Z}$ to first order in $b$ is an easy task with the diagrammatic rules given in Sect. 2 . The only contributing diagram is given in Fig. 8. Then $\mathscr{Z}$ reads

$$
\begin{aligned}
\mathscr{Z} & =1-b \int \frac{d^{d} q_{1}}{(2 \pi)^{d}} \ldots \frac{d^{d} q_{P-1}}{(2 \pi)^{d}} \int_{0}^{s_{1}} d s_{1} \ldots \int_{0}^{s_{P}} d s_{P} e^{-\frac{1}{2} q_{1}^{2} s_{1}} e^{-\frac{1}{2}\left(q_{1}+q_{2}\right)^{2} s_{2}} \ldots e^{-\frac{1}{2} q_{P-1}^{2} s_{P}} \\
& =1-b(2 \pi)^{-\frac{d}{2}(P-1)} \int_{0}^{\left\{S_{1}\right\}} \int_{0}^{P} \prod_{1}^{P} d s_{i}(\operatorname{det} A)^{-d / 2}
\end{aligned}
$$

where the determinant of the Gaussian integration is

$$
\operatorname{det} A=\left(\sum_{1}^{P} \frac{1}{s_{i}}\right) \prod_{1}^{P} s_{i} .
$$

The integral (4.6) can be diverging at the origin when all $\left\{s_{i}\right\}$ go to zero together. This happens only when $d \geqq d^{*}=2 P /(P-1)$. We shall search here the dominant pole when $d \rightarrow d^{*-}$, setting

$$
d=d^{*}-\varepsilon=\frac{2 P}{P-1}-\varepsilon, \quad \varepsilon>0
$$

Hence the dimensionless parameter $z$ (4.2) reads

$$
z=(2 \pi)^{-\frac{d}{2}(P-1)} b S^{\varepsilon(P-1) / 2} .
$$

To integrate (4.6), we perform the change of variables

with

$$
s_{i}=S u_{i}^{-1}, \quad i=1, \ldots, P,
$$

$$
S_{i}=\sigma_{i} S
$$

$S$ gives thus the common scale of areas $\left\{S_{i}\right\}$. Hence we can write, using (4.2),

$$
\begin{gathered}
\mathscr{Z}=1-z \mathscr{I}, \\
\mathscr{I}=\int_{\left\{\sigma_{i}^{-1}\right\}}^{\infty} \ldots \int_{1}^{\infty} \prod_{1}^{P} d u_{i} u_{i}^{d / 2-2}\left(\sum_{1}^{P} u_{i}\right)^{-d / 2} .
\end{gathered}
$$


It will be convenient to decouple the $u_{i}$ 's by using the integral representation

$$
\mathscr{I}=\frac{1}{\Gamma(d / 2)} \int_{0}^{\infty} d p p^{\frac{d}{2}-1} \prod_{i=1}^{P} F\left(p, \sigma_{i}^{-1}\right)
$$

where $F$ reads

$$
\begin{aligned}
F\left(p, \sigma^{-1}\right) & =\int_{\sigma^{-1}}^{\infty} d u u^{\frac{d}{2}-2} e^{-p u} \\
& =p^{1-\frac{d}{2}} \Gamma\left(\frac{d}{2}-1, p \sigma^{-1}\right)
\end{aligned}
$$

in terms of the incomplete gamma function:

$$
\Gamma(\alpha, x)=\int_{x}^{\infty} e^{-t} t^{\alpha-1} d t
$$

Now, recalling that the possible divergence in (4.6) arrive when $\{s\} \rightarrow 0$, hence $\{u\} \rightarrow \infty$, we conclude that in the $p$ representation they will occur for $p \rightarrow 0$. Hence, to obtain the dominant pole in (4.6) we may as well set $\sigma_{i}=\infty$ in (4.13), provided that we regularize at large $p$ integral (4.12), by, e.g., a supplementary factor $e^{-p}$. Hence, the following equality holds true at the dominant diverging order when $d \rightarrow d^{*}$ :

$$
\begin{aligned}
\mathscr{I} & =\mathscr{I}_{\text {sing }}+O(1) \\
\mathscr{I}_{\text {sing }} & =\frac{1}{\Gamma(d / 2)} \int_{0}^{\infty} d p^{\prime} p^{\prime} \frac{d}{2}-1+P\left(1-\frac{d}{2}\right) e^{-p^{\prime}} \times \Gamma^{P}\left(\frac{d}{2}-1\right) \\
& =\frac{\Gamma^{P}\left(\frac{d}{2}-1\right)}{\Gamma\left(\frac{d}{2}\right)} \Gamma\left(P-\frac{d}{2}(P-1)\right) .
\end{aligned}
$$

We perform the $\varepsilon$ expansion of this result [using (4.8)] and find the singular part of $\mathscr{I}$

$$
\mathscr{I}_{\text {sing }}=\Gamma^{P-1}\left(\frac{1}{P-1}\right) \frac{2}{\varepsilon}+\ldots
$$

Hence, the partition function (4.5) has finally the first order expansion

$$
\mathscr{Z}=1-z \frac{2}{\varepsilon} \Gamma^{P-1}\left(\frac{1}{P-1}\right)+\ldots .
$$

We note that the dominant $\frac{1}{\varepsilon}$ terms does not depend on the distribution $\left\{S_{i}\right\}$, but only on its common scale $S$ through $z$. As a consequence, the dominant scaling behaviour of $\mathscr{Z}$, to be determined now, will depend only on $S$. One can take for instance all areas equal $S_{i}=S$, or equally well $S_{1}=S, S_{i \neq 1}=\infty$. 


\section{4.b. Renormalization}

The method is a generalization of those of Sects. 2 and 3, and has not been presented before. We use a renormalized coupling constant, here the $P^{\text {th }}$ dimensionless virial coefficient $g$, which will reach a fixed point value for $d<d^{*}$. We define $g$ as

where

$$
g=\mathscr{Z}_{P}(S, \ldots, S)(2 \pi S)^{-(P-1) d / 2},
$$

$$
\mathscr{Z}_{P}(S, \ldots, S) \equiv b\left\langle\int_{0}^{S} \ldots \int_{0}^{S} \prod_{i=1}^{P} d s_{i} \prod_{i=1}^{P-1} \delta\left[\mathbf{r}_{i}\left(s_{i}\right)-\mathbf{r}_{i+1}\left(s_{i+1}\right)\right] \delta^{d}\left[\mathbf{r}_{1}(0)\right]\right\rangle
$$

describes the connected partition function of $P$ walks of lenghts $S$, whose trajectories have at least one point in common, and with one origin fixed to eliminate the trivial translational invariance. The second factor in (4.16) makes $g$ dimensionless, as can be checked from (4.17). $\mathscr{Z}_{P}$ is easily evaluated to second order with the help of Fig. 9:

$$
\mathscr{Z}_{P}=b S^{P}-b^{2}(2 \pi)^{-(P-1) d / 2} 2^{P-1} \int_{0}^{S} \ldots \int_{0}^{S} \prod_{i=1}^{P} d s_{i} \prod_{i=1}^{P}\left(S-s_{i}\right)\left[\left(\sum_{i=1}^{P} \frac{1}{s_{i}}\right) \prod_{i=1}^{P} s_{i}\right]^{-d / 2} .
$$

The factor $2^{P-1}$ comes from all the equivalent diagrams obtained by twisting $P-1$ walks in Fig. 9. Now, the singular part in (4.18) is given by the neighborhood of the origin, and is therefore the same as in multiple integral (4.6), (4.11). We may write immediately to this order

$$
\mathscr{Z}_{P}=b S^{P}\left[1-z 2^{P-1} \mathscr{I}_{\text {sing }}\right],
$$

where $\mathscr{I}_{\text {sing }}$ is given explicitly by Eq. (4.14). Hence $g$ (4.16) finally reads

with

$$
g=z\left(1-z \mathscr{I}^{\prime} \frac{2}{\varepsilon}\right)
$$

$$
\mathscr{I}^{\prime}=2^{P-1} \Gamma^{P-1}\left(\frac{1}{P-1}\right)
$$

Its Wilson function is defined as usual by

$$
W[g, \varepsilon]=\left.S \frac{\partial}{\partial S} g\right|_{b, \varepsilon}=\left.\frac{\varepsilon}{2}(P-1) z \frac{\partial}{\partial z} g\right|_{\varepsilon},
$$
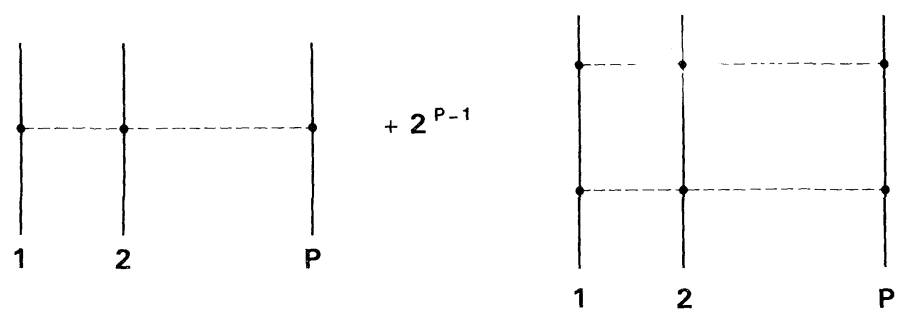

Fig. 9. Diagrams for the connected partition functions $\mathscr{Z}_{P}(S, \ldots, S)$ of $P$ paths 
and reads from (4.19) in terms of $g$

$$
W[g, \varepsilon]=(P-1)\left(\frac{\varepsilon}{2} g-g^{2} \mathscr{I}^{\prime}+O\left(g^{3}\right)\right) .
$$

$W$ is regular when $\varepsilon \rightarrow 0$, to all orders in $g$. For $\varepsilon \neq 0$, the fixed point value of $g$ is thus obtained as

$$
g^{*}=\frac{\varepsilon}{2^{P}} \Gamma^{1-P}\left(\frac{1}{P-1}\right)+O\left(\varepsilon^{2}\right)
$$

At the critical dimension $d^{*}, \varepsilon=0$, we use the regular Wilson function for $P$ walks

$$
W[g, 0]=-g^{2}(P-1) \mathscr{I}^{\prime}+O\left(g^{3}\right) .
$$

This yields by integration the asymptotic behaviour of $g$,

$$
\begin{aligned}
g & =\left[(P-1) \mathscr{I}^{\prime} \ln S\right]^{-1}+O\left(\ln \ln S / \ln ^{2} S\right) \\
& =2^{1-P} \Gamma^{1-P}\left(\frac{1}{P-1}\right)[(P-1) \ln S]^{-1}+O\left(\ln \ln S / \ln ^{2} S\right) .
\end{aligned}
$$

It remains to evaluate the asymptotic behaviour of partition function $\mathscr{Z}$ (4.15). For $S$ läge and $d<d^{*}$, we expect it to scale like $\mathscr{Z} \sim S^{\zeta_{P}}$, where $\zeta_{P}$ is a universal critical exponent characterizing the simulatneous intersection of $P$ walks. As in Sect. 2.3, we define an associated regular scaling function

$$
\zeta_{P}[g, \varepsilon]=S \frac{\partial}{\partial S} \ln \mathscr{Z}
$$

According to (4.15), it reads simply

$$
\zeta_{P}[g, \varepsilon]=-\frac{P-1}{2} \varepsilon \mathscr{I} g+O\left(g^{2}\right)=-(P-1) \Gamma^{P-1}\left(\frac{1}{P-1}\right) g+O\left(g^{2}\right) .
$$

Therefore its fixed point value is obtained for $d<d^{*}$ from (4.22),

$$
\zeta_{P}=-\frac{\varepsilon}{2^{P}}(P-1)+O\left(\varepsilon^{2}\right)
$$

For $P=2,3$ we recover (2.25 bis), (3.24), as it must.

At the critical dimension $d=d^{*}$, we insert in (4.24), (4.25) the form (4.23) for $g$ :

$$
\mathrm{S} \frac{\partial}{\partial S} \ln \mathscr{Z}=-1 /\left(2^{P-1} \ln S\right)+\ldots
$$

hence

$$
\mathscr{Z} \approx(\ln S)^{-1 / 2 P-1}
$$

This gives the generalisation to $P$ walks of results (2.26), (3.25) for $P=2,3$, and is valid in a space of continuous dimension $d^{*}=2 P /(P-1)$. 
If we look instead at the probability $\mathscr{Z}^{\prime}$ that one walk of length $S$ does not intersect $P-1$ two-sided infinite walks (Fig. 8b), we simply get an extra-factor $2^{P-1}$ in the new partition function $\mathscr{Z}^{\prime}$, obtained from $(4.5),(4.15)$ as

$$
\mathscr{Z}^{\prime}=1-z \mathscr{I} 2^{P-1} \text {. }
$$

This corresponds, for each two-sided walk, to the choice of interaction with the positive time or negative time side. Immediately the critical exponent $\zeta_{P}^{\prime}$ such that $\mathscr{Z}^{\prime} \sim S^{\zeta_{P}^{\prime}}$ becomes, instead of $\zeta_{P}(4.26)$,

$$
\zeta_{P}^{\prime}=-\frac{\varepsilon}{2}(P-1)+O\left(\varepsilon^{2}\right) .
$$

At the critical dimension $d^{*}$ :

$$
\mathscr{Z}^{\prime} \approx(\ln S)^{-1}
$$

as already found for $P=2,3$.

We can also consider probabilities of non-empty intersections, which can be evaluated in a way entirely similar to that of Sect. 2.3. In particular, one can evaluate explicitly the analogue of probability $\mathscr{Z}_{e}(2.41)$ and $\mathscr{Z}_{f}(3.40),(3.44),(3.45)$, where one finite walk starts at $\mathbf{x}_{0}$ while $P-1$ infinite ones start at $\mathbf{0}$. But we shall not pursue this question here.

It would be interesting to derive the results (4.27), (4.29) from a probabilistic point of view, which would require to work in $\mathbb{Z}^{d}$, with $d \notin \mathbb{N}$.

\section{More Results Near Four Dimensions}

In Sect. 2, we have considered the intersection properties of two random walks, for which $d=4$ is the critical dimension. However, we have contented ourselves there with a simple one-loop calculation, which reproduced in a simple way probabilistic results. The renormalization process appeared at this order as an almost trivial statement, and perhaps its essential tool, i.e. substituting $g$ to $z$, did not show its actual efficiency. So, we shall here go one step further and calculate the probabilities of intersection to second order in $\varepsilon$ for $d=4-\varepsilon$, and in $d=4$ calculate sub-dominant logarithmic terms, as we did in ref. [20] for polymers.

\section{5.a. Two Walks to $O\left(\varepsilon^{2}\right)$}

Let us first consider the probability $\mathscr{Z}(2.6)$ that two walks starting at $\mathbf{0}$ in $\mathbb{Z}^{d}$ or $\mathbb{R}^{d}$, do not intersect each other before length $S$ :

$$
\mathscr{Z}=\left\langle\delta^{d}\left[\mathbf{r}_{1}(0)\right] \delta^{d}\left[\mathbf{r}_{2}(0)\right]\right\rangle,
$$

where the average is taken with respect to Edwards' generalized weight (2.1). The expansion of $\mathscr{Z}$ to second order in $b$ is given by the diagrams of Fig. 1 and Fig. 10. It reads

$$
\mathscr{Z}=1+\mathscr{I}+\mathscr{I}_{1}+\mathscr{I}_{2}
$$




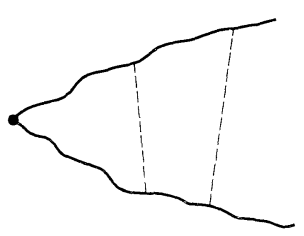

(a)

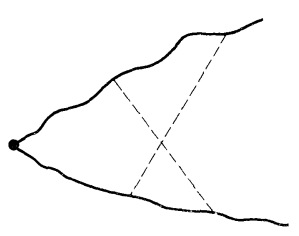

(b)

Fig. 10a and b. Second order diagrams involved in the probability $\mathscr{Z}(S, S)$ that two Brownian paths do not intersect, except at their common origin, at order $O\left(\varepsilon^{2}\right), d=4-\varepsilon$. Their contributions are a $\mathscr{I}_{1}(5.7)$ and $\mathbf{b} \mathscr{I}_{2}(5.8)$

where $\mathscr{I}$ has been calculated in Sect. 2, Eq. (2.14),

$$
\mathscr{I}=-z \frac{1}{(1-d / 2)(2-d / 2)}\left[2^{2-d / 2}-2\right] .
$$

$\mathscr{I}_{1}\left(\mathscr{I}_{2}\right)$ correspond respectively to Fig. 10 a, b. They read explicitly

$$
\begin{gathered}
\mathscr{I}_{1}=z^{2} \int_{0}^{\infty} d s_{1} d s_{2} d s_{1}^{\prime} d s_{2}^{\prime} \theta\left(1-s_{1}-s_{2}\right) \theta\left(1-s_{1}^{\prime}-s_{2}^{\prime}\right)\left(s_{1}+s_{1}^{\prime}\right)^{-d / 2}\left(s_{2}+s_{2}^{\prime}\right)^{-d / 2}, \\
\mathscr{I}_{2}=z^{2} \int_{0}^{\infty} d s_{1} d s_{2} d s_{1}^{\prime} d s_{2}^{\prime} \theta\left(1-s_{1}-s_{2}\right) \theta\left(1-s_{1}^{\prime}-s_{2}^{\prime}\right)\left[\left(s_{1}+s_{1}^{\prime}\right)\left(s_{2}+s_{2}^{\prime}\right)+s_{2} s_{2}^{\prime}\right]^{-d / 2} .
\end{gathered}
$$

Now, the actual scaling behaviour of $\mathscr{Z}$ will be determined by the Laurent expansion of these integrals when $d \rightarrow 4$. When have for $d=4-\varepsilon$,

$$
\mathscr{I}=z\left(-\frac{2}{\varepsilon}-1+\ln 2\right) .
$$

The evaluation of $\mathscr{I}_{1}(5.4)$ is not really difficult and yields

$$
\mathscr{I}_{1}=z^{2} 4\left[\frac{1}{\varepsilon^{2}}+\frac{1}{\varepsilon}(1-\ln 2)\right] .
$$

The last evaluation $\mathscr{I}_{2}$ is not simple [43]. We find

$$
\mathscr{I}_{2}=z^{2}\left[\frac{2}{\varepsilon^{2}}+\frac{1}{\varepsilon}(3-2 \ln 2)\right] .
$$

(Note that the calculations are performed here for walks of finite and fixed lengths $S$. This amounts in field theory to calculate diagrams involving two different masses, which are related by Laplace transform to polymer diagrams with fixed lengths $S$. This procedure is more complicated and different from the ones used in $[10,44]$, where the total length $S_{1}+S_{2}$ of the walks fluctuates with a standard killing 
factor $e^{-m^{2}\left(S_{1}+S_{2}\right)}$.) Hence we find

$$
\mathscr{Z}=1+z\left(-\frac{2}{\varepsilon}-1+\ln 2\right)+z^{2}\left[\frac{6}{\varepsilon^{2}}+\frac{1}{\varepsilon}(7-6 \ln 2)\right]+\ldots .
$$

We need now to renormalize the theory at this order.

\section{5.b. Renormalization}

We need to know the dimensionless "second virial coefficient" $g$ or "intersection local time" (2.16) to third order in $z$. The diagrams contributing to the connected partition function $(2.17) \mathscr{Z}_{2}(S, S)$ to third order in $b$ are given on Fig. 11, together with their respective contributions and combinatorial weights. They can be calculated by the same method as $\mathscr{Z}$ above. (See also $[18,22]$, the first diagram of Fig. 10 in [18] being corrected here.) We have

$$
\mathscr{Z}_{2}(S, S)=b S^{2}\left\{1-z\left(\frac{4}{\varepsilon}+1-4 \ln 2\right)+z^{2} \frac{1}{\varepsilon^{2}}[16+\varepsilon(12-32 \ln 2)]\right\},
$$

and $g(2.16)$ reads at this order

$$
g=z+z^{2}\left(-\frac{4}{\varepsilon}-1+4 \ln 2\right)+z^{3} \frac{1}{\varepsilon^{2}}[16+\varepsilon(12-32 \ln 2)]
$$

in agreement with some results of [22]. This equation is inverted to the same order into

$$
z=g+g^{2}\left(\frac{4}{\varepsilon}+1-4 \ln 2\right)+g^{3} \frac{1}{\varepsilon^{2}}[16+\varepsilon(4-32 \ln 2)] .
$$

The Wilson function (2.20) associated with $g$ reads now

$$
W=\left.\frac{\varepsilon}{2} z \frac{\partial}{\partial z} g\right|_{\varepsilon}=\frac{\varepsilon}{2} z+z^{2}[-4+\varepsilon(-1+4 \ln 2)]+z^{3} \frac{1}{\varepsilon}[24+\varepsilon(18-48 \ln 2)] \text {. }
$$

While being singular in terms of $z$ when $\varepsilon \rightarrow 0$, it is regular in terms of $g, \varepsilon$ after subsitution of (5.12), as it must:

$$
\begin{aligned}
& W[g, \varepsilon]=\frac{\varepsilon}{2} g+\left[-2+\varepsilon\left(-\frac{1}{2}+2 \ln 2\right)\right] g^{2}+4 g^{3}+O\left(g^{4}\right) . \\
& -\left(\frac{4}{\varepsilon}+1-4 \ln 2\right)
\end{aligned}
$$
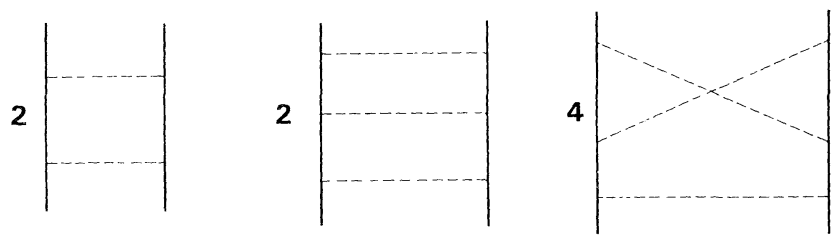

Fig. 11. Second order contributions to $\mathscr{Z}_{2}(S, S)$ and their combinatorial weights, resulting in $(5.10)$ 
$W$ corresponds to the intersection properties of two Brownian paths or two random walks in $d=4-\varepsilon$ dimensions. It is similar, but of course different, from the one associated with intersections of two polymers or self-avoiding walks (see [18]). The fixed point value of $g$ such that $W\left[g^{*}, \varepsilon\right]=0$ is therefore in $d<4$,

$$
g^{*}=\frac{\varepsilon}{4}+\left(\frac{\varepsilon}{4}\right)^{2}(1+4 \ln 2)
$$

For $d=4$, we set $\varepsilon=0$ into (5.13) and get the regular differential equation

$$
W[g, \varepsilon=0]=S \frac{\partial}{\partial S} g=-2 g^{2}+4 g^{3}+O\left(g^{4}\right) .
$$

This simple equation describes essentially the way the intersection local time (2.17) changes with the "times" $S$ allowed to the random walks in $\mathbb{Z}^{4}$. Note that its analogue for usual polymers in $d=4$ reads [20] $S \frac{\partial}{\partial S} g=-4 g^{2}+17 g^{3}$, which shows the difference of intersection properties for Brownian and self-avoiding walks. Equation (5.15) is integrated into

$$
\ln \left(S / s_{0}\right)=\frac{1}{2 g}-\ln g+O(g)
$$

where $s_{0}$ is an integration constant, $g$ being small. We therefore find

$$
g=\frac{1}{2 \ln \left(S / s_{0}\right)}\left\{1+\frac{\ln \left[2 \ln \left(S / s_{0}\right)\right]}{\ln \left(S / s_{0}\right)}+O\left[\frac{\ln ^{2} \ln \left(S / s_{0}\right)}{\ln ^{2}\left(S / s_{0}\right)}, \ln ^{-2}\left(S / s_{0}\right)\right]\right\} .
$$

(Note that a change of integration constant $s_{0}$ brings in terms of order $\ln ^{-2} S$ in $g$. Hence $s_{0}$ can also be seen [20] as the value such that there are no such terms in $g$.) We may write in short for simplicity

$$
g=\frac{1}{2 \ln S}\left(1+\frac{\ln \ln S}{\ln S}+\ldots\right)
$$

\section{5.c. Asymptotic Evaluation of the Probability of no Intersection}

We calculate the finite scaling function $\zeta(2.24)$ associated with $\mathscr{Z}$, from expansion (5.9):

$$
\zeta=\frac{\varepsilon}{2} z \frac{\partial}{\partial z} \ln \mathscr{Z}=z\left[-1+\frac{\varepsilon}{2}(-1+\ln 2)\right]+z^{2}\left(\frac{4}{\varepsilon}+5-4 \ln 2\right) .
$$

Substituting (5.12) we find the regular scaling function

$$
\zeta[g, \varepsilon]=g\left[-1+(-1+\ln 2) \frac{\varepsilon}{2}\right]+g^{2}(2+2 \ln 2) .
$$

In less than four dimensions, its fixed point value gives the universal exponent $\zeta$,

$$
\zeta=\zeta\left[g^{*}, \varepsilon\right]=-\frac{\varepsilon}{4}-\left(\frac{\varepsilon}{4}\right)^{2}+O\left(\varepsilon^{3}\right)
$$


such that

$$
\mathscr{Z} \sim S^{\zeta} \text { for } S \rightarrow \infty
$$

$\mathscr{Z}$ being the probability that two Brownian paths in $\mathbb{R}^{d}, d<4$, starting at the origin, do not intersect again before "time" $S$. For $d=3, \varepsilon=1$, we find an approximate value $\zeta \approx-\frac{5}{16} \simeq-0.31$. It would be most interesting to try and determine its exact value in two dimensions, using conformal invariance and Coulomb gas methods [45]. In $d=4$, we calculate the scaling function (5.18) at $\varepsilon=0$,

$$
\zeta[g, 0]=-g+(2+2 \ln 2) g^{2}+O\left(g^{3}\right) .
$$

The best way [20] to evaluate $\mathscr{Z}$ is to use definitions (2.20), (2.24) to write

$$
\left.\frac{\partial}{\partial g} \ln \mathscr{Z}\right|_{d=4}=\zeta[g, 0] / W[g, 0],
$$

and obtain from (5.20), (5.15) the expansion

Hence

$$
\frac{\partial}{\partial g} \ln \mathscr{Z}=\frac{1}{2 g}-\ln 2+O(g)
$$

$$
\mathscr{Z}=C g^{1 / 2}\left[1-g \ln 2+O\left(g^{2}\right)\right],
$$

where $C$ is an integration constant, which will be model dependent [20] and thus not universal. Actually, it can be calculated in terms of $b$ for the continuum model (2.1) [20]. Note that the value (5.21), together with our result (5.17), refines considerably Lawler's estimation (2.26) in four dimensions. If we do not take into account the unknown $s_{1}$, in $g$, we may use $(5.17 \mathrm{bis})$ and write the simplified universal result in $d=4$,

$$
\mathscr{Z}=C\left(\frac{1}{2 \ln S}\right)^{1 / 2}\left\{1+\frac{1}{2} \frac{\ln \ln S}{\ln S}+O\left(\ln ^{-1} S\right)\right\} .
$$

If however one knew the value of $s_{0}$, one could write the better asymptotic estimate

$$
\begin{aligned}
\mathscr{Z} & =C\left(\frac{1}{2 \ln \left(S / s_{0}\right)}\right)^{1 / 2}\left\{1+\frac{1}{2} \frac{\ln \left(2 \ln \left(S / s_{0}\right)\right)}{\ln \left(S / s_{0}\right)}-\frac{\ln 2}{2 \ln \left(S / s_{0}\right)}+O\left(\frac{\ln ^{2} \ln S}{\ln ^{2} S}\right)\right\} \\
& =C\left(\frac{1}{2 \ln \left(S / s_{0}\right)}\right)^{1 / 2}\left\{1+\frac{1}{2} \frac{\ln \ln \left(S / s_{0}\right)}{\ln \left(S / s_{0}\right)}+O\left(\frac{\ln ^{2} \ln S}{\ln ^{2} S}\right)\right\} .
\end{aligned}
$$

We can also apply these results to the other geometrical cases described in Sect. (2.d) (Fig. 4). We shall not need the second order contributions to these quantities. If one uses indeed expansion (5.17 bis) for $g$, as we have seen in Eqs. (5.21)--(5.23), the correction terms of order $O(g)$ are required only if $s_{0}$ is known, while the $\frac{\ln \ln S}{\ln S}$ corrections terms are automatically brought in by $g$ itself $(5.17 \mathrm{bis})$. So we ameliorate directly Lawler's results of Sect. (2.d) into 
Case a (2.35)

$$
q(n) \rightarrow \mathscr{Z}(S, \infty)=C^{\prime}(\ln S)^{-1 / 2}\left(1+\frac{\ln \ln S}{2 \ln S}+\ldots\right),
$$

where $C^{\prime}$ is a non-universal constant.

Case b (2.36)

$$
\mathscr{Z}(S,-\infty,+\infty)=C^{\prime \prime}(\ln S)^{-1}\left(1+\frac{\ln \ln S}{\ln S}+\ldots\right),
$$

where $C^{\prime \prime}$ is non-universal.

Caxe c (2.39)

$$
\mathscr{Z}_{c}=\left(\frac{1}{2 \ln S}+\frac{1}{2 \ln ^{2} S} \ln \ln S+\ldots\right) \ln (\beta / \alpha)
$$

Case $d$ (2.40 bis)

$$
\mathscr{Z}_{d}=\left(\frac{1}{2 \ln S}+\frac{1}{2 \ln ^{2} S} \ln \ln S+\ldots\right) \ln (1+1 / \alpha) .
$$

Case e (2.41 bis)

$$
\mathscr{Z}_{e}=\left(\frac{1}{2 \ln S}+\frac{1}{2 \ln ^{2} S} \ln \ln S+\ldots\right)\left[\frac{1}{\alpha}\left(1-e^{-\alpha}\right)-E_{i}(-\alpha)\right] .
$$

\section{Nets of Walks}

In this last section we shall give a full generalization of the intersection problem of random walks in $\mathbb{R}^{d}$ or $\mathbb{Z}^{d}$. This generalisation seems not to have been considered previously in the mathematics literature. It is inspired from known results in the theory of polymers, where we considered [45] networks of fixed topology, and calculated their critical properties. Some similar situations for walks in one dimension (e.g. the "vicious drunk" problem in [46]) have also been considered, in the context of wetting and melting phenomena [46] and commensurateincommensurate transitions [47].

Let us consider in $\mathbb{Z}^{d}$ or $\mathbb{R}^{d}, \mathcal{N}$ independent simple random walks or Brownian paths. We constrain these walks to meet at some intersection points of degrees $L, L$ $\geqq 1$, where $L$ walks meet together. $L=1$ corresponds to free ends or origins of some walks (Fig. 12). The location of the vertices is not fixed in space, but the topology of the net of walks $\mathscr{G}$ so formed is arbitrary but fixed [45]. This is entirely reminiscent of gels, or branched polymers, i.e. polymer networks, with fixed topology. For simplicity, we assume all the walks to have the same length $S$ between the vertices, but arbitrary lengths could be considered as well. The topology of $\mathscr{G}$ will be characterized by the prescribed set of numbers $n_{L}$ of crossing points, or vertices, of type $L$ where $L$ walks meet together in the net of walks $\mathscr{G}$. The set $\left\{n_{L}\right\}$ does not entirely define the topology of the net, but [45] it is the only relevant parameter set for the scaling properties of the probabilities of intersections, as we shall see. We 


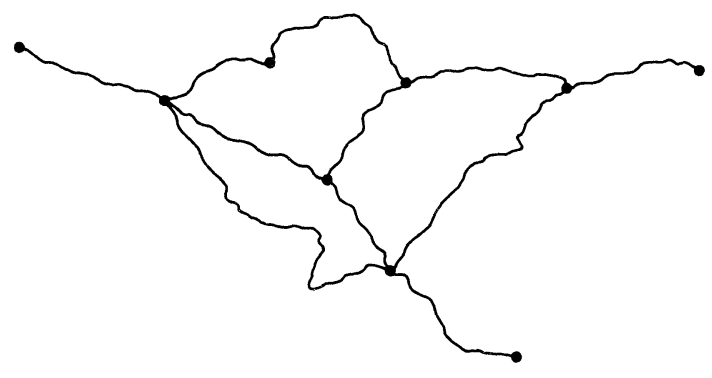

$$
\begin{gathered}
n_{1}=3, \quad n_{2}=1, \quad n_{3}=3, \quad n_{4}=2 \\
Q N=11
\end{gathered}
$$

Fig. 12. A net of $\mathcal{N}=11$ random walks of the same lengths, constrained to intersect at their extremities at $n_{L} L$-line vertices, with $n_{1}=3$ single origins or extremities of walks, $n_{2}=1$ double point, $n_{3}=3$ triple points, $n_{4}=2$ quadruple points. Outside these vertices, the walks do not intersect one another

now ask: What is the relative probability $\mathscr{Z}(\mathscr{G})$ that the $\mathcal{N}$ random walks constrained to meet at the vertices of $\mathscr{G}$ at "time" $S$, never cross one another before "time" $S$ ?

This is obviously a full generalization of Lawler's probabilities for two independent random walks starting at $\mathbf{0}$. We note that the latter case is simply described by the set

$$
\left\{n_{L}\right\}=\left\{n_{1}=2, n_{2}=1, n_{L \geqq 3}=0\right\},
$$

since there are $2=n_{1}$ free ends of walks, and $1=n_{2}$ double point at the origin.

For a general network $\mathscr{G}$ made of $n_{L} L$-leg vertices, the total numbers of walks $\mathscr{N}$ and of independent loops $\mathscr{L}$ in the net $\mathscr{G}$ are given by the topological relations

$$
\mathscr{N}=\sum_{L \geqq 1} \frac{1}{2} L n_{L}, \quad \mathscr{L}=\sum_{L \geqq 1} \frac{1}{2}(L-2) n_{L}+1 .
$$

In [45] we have given the basic renormalization theory required for dealing with any polymer network. Here we shall adapt it to any net of walks, where it is even simpler.

\section{6.a. Continuum Theory}

For describing the mutual avoidance of the $\mathscr{N}$ walks, it is sufficient to generalize the Edwards' model (2.1) to the weight $\mathscr{P}_{\mathscr{N}}$ of $\mathscr{N}$ walks of lengths $S$, interacting via $\left(\begin{array}{c}\mathscr{N} \\ 2\end{array}\right)$ two-body $b \delta^{d}$ interactions. The critical dimension remains $d=4$. The probability of no further crossings $\mathscr{Z}(\mathscr{G})(6.1)$ is simply given by a functional integral over all configurations, respecting the topological constraint of forming $n_{L}$ $L$-leg intersection points [45]:

$$
\mathscr{Z}(\mathscr{G})=\int \prod_{i=1}^{\mathscr{N}} d\left\{\mathbf{r}_{i}\right\} \mathscr{P}_{\mathscr{N}}\left\{\mathbf{r}_{i}\right\} \delta^{d}(\mathscr{G}) / \int \prod_{i=1}^{\mathscr{N}} d\left\{\mathbf{r}_{i}\right\} P_{0}\left\{\mathbf{r}_{i}\right\} \prod_{i=1}^{\mathscr{N}} \delta^{d}\left[\mathbf{r}_{i}(0)\right] .
$$


$\delta^{d}(\mathscr{G})$ is symbolic : it is the product of all necessary $\delta^{d}$ distributions in $\mathbb{R}^{d}$, connecting the walks in the net $\mathscr{G}$, plus one for eliminating translational invariance. The total number of $\delta^{d}$ distributions in $\delta(\mathscr{G})$ is thus [45]

$$
\Delta=\sum_{L \geqq 1}(L-1) n_{L}+1 \text {. }
$$

$\mathscr{Z}(\mathscr{G})$ can be calculated by dimensional regularization. Then, by dimensional analysis, it can be written as

$$
\mathscr{Z}(\mathscr{G})=(2 \pi S)^{(\mathscr{N}-\Delta) d / 2} Z_{\mathscr{G}}(z, d),
$$

where $Z_{\mathscr{G}}$ is a dimensionless reduced partition function, depending only on $z$ (2.3) and on space dimension $d$. From (6.3), (6.5) we get the "naive" scaling dimension of $\mathscr{Z}(\mathscr{G})$

Hence we find

$$
(\mathscr{N}-\Delta) d / 2=-\mathscr{L} d / 2
$$

$$
\mathscr{Z}(\mathscr{G})=(2 \pi S)^{-\mathscr{L} d / 2} Z_{\mathscr{G}}(z, d) .
$$

Note that the first factor has been chosen to be precisely the exact Brownian scale of the probability for $b=0$ or $z=0$. More precisely, the exact Brownian value of $\mathscr{Z}(\mathscr{G})$ can be shown to be

$$
\mathscr{Z}^{B}(\mathscr{G})=(2 \pi S)^{-\mathscr{L} d / 2}\left(\operatorname{det} \mathbb{C}_{\mathscr{G} /\{a\}}\right)^{-d / 2},
$$

where $\mathbb{C}_{\mathscr{G}}$ is the connexion matrix of the graph $\mathscr{G}$. It is a $\mathscr{V} \times \mathscr{V}$ matrix, where $\mathscr{V}=\sum_{L \geqq 1} n_{L}$ is the total number of vertices of $\mathscr{G}$. The elements $\mathbb{C}_{a b}$ are labelled by the vertices of $\mathscr{G}$ and are defined as

$$
\begin{array}{ll}
a \neq b, & \mathbb{C}_{a b}=-\# \text { lines joining } a \text { to } b \\
a=b, & \mathbb{C}_{a a}=-\sum_{b \neq a} \mathbb{C}_{a b}=\# \text { lines attached to } a \text { by one (and only one) } \\
\text { extremitity. }
\end{array}
$$

This matrix has a zero mode, hence $\operatorname{det} \mathbb{C}_{\mathscr{G}}=0$. The notation $\operatorname{det} \mathbb{C}_{\mathscr{G} /\{a\}}$ above is then the minor of $\mathbb{C}_{\mathscr{G}}$ with respect to any vertex $a$ of $\mathscr{G}$, and is independent of $a$.

\section{6.b. Renormalization by Star Walks}

Applying the results of [45], we now state the following multiplicative structure of the renormalization of $Z_{\mathscr{G}}$. This dimensionless partition function possesses poles in $1 / \varepsilon$ to all orders in its perturbation series in terms of $z$ [see for instance $\mathscr{Z}$ in Eq. (5.9)]. For extracting them it is sufficient to consider the partition function $\mathscr{Z}\left(\mathscr{S}_{L}\right)$ of a single star walk $\mathscr{S}_{L}$, made of $L$ identical mutually avoiding walks of lengths $S$, all starting at the origin (Fig. 13). For this star $\mathscr{S}_{L}$ there is no constitutive loop and Eq. (6.8) shows that $\mathscr{Z}\left(\mathscr{S}_{L}\right)$ is dimensionless:

$$
\mathscr{Z}\left(\mathscr{S}_{L}\right) \equiv Z_{\mathscr{S}_{L}}(z, d)
$$

Furthermore, since a single walk has no other walk to avoid, one has trivially

$$
\mathscr{Z}\left(\mathscr{S}_{1}\right) \equiv 1
$$



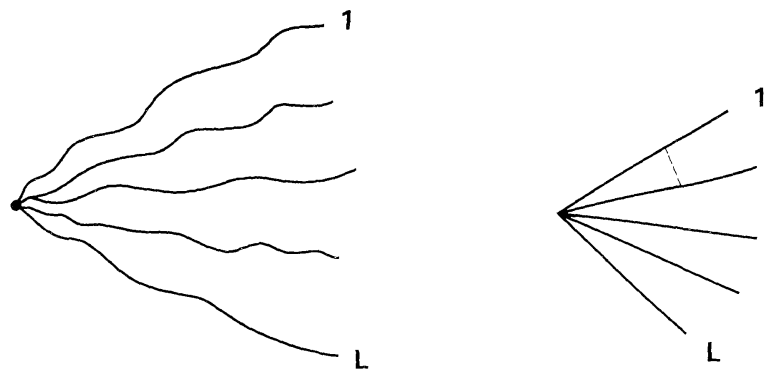

Fig. 13. A star walk $\mathscr{P}_{L}$ made of $L$ independent random walks issued from the same point, and the one-loop diagram contributing to the probability $\mathscr{Z}\left(\mathscr{P}_{L}\right)(6.18)$ of no intersection, calculated from continuum weight $(2.1)$

Now, as shown in [45], for a general network $\mathscr{G}$, we may factorize $\mathscr{Z}(\mathscr{G})$ into

$$
\mathscr{Z}(\mathscr{G})=(2 \pi S)^{-\mathscr{L} d / 2} \prod_{L \geqq 1}\left(\hat{Z}_{L}\right)^{n_{L}} A_{\mathscr{G}}(z, d)
$$

where [45]

$$
\hat{Z}_{L}(z, d) \equiv \mathscr{Z}\left(\mathscr{S}_{L}\right) \mathscr{Z}^{-L / 2}\left(\mathscr{S}_{1}\right)
$$

and where the amplitude $A_{\mathscr{G}}(z, d)$ reaches a finite fixed point value when $z \rightarrow \infty$,

$$
A_{\mathscr{G}}(z, d) \rightarrow A_{\mathscr{G}}^{*}, \quad z \rightarrow \infty, \quad d<4 .
$$

Note that the above definitions are such that $A_{\mathscr{G}}(z=0, d) \equiv 1$. The amplitude $A_{\mathscr{G}}(z, d)$ becomes pole free in $\varepsilon$ to all orders in terms of $g$, when the latter $g(2.16)$ is substituted to $z$. Note also that necessarily

$$
\left(\operatorname{det} \mathbb{C}_{\mathscr{G} /\{a\}}\right)^{-d / 2} .
$$

[Note that in principle $\left[45,18\right.$ ], in Eq. (6.11) one must also replace $S^{-d \mathscr{S} / 2}$, by $R^{-d \mathscr{S}}$, where $R$ is the physical size (2.2) of the isolated walk when the latter is selfavoiding. Here there is no renormalization of this kind, since the simple random walks are not interacting with themselves. In field theory, there is here no mass renormalization.]

The meaning of the multiplicative renormalization equation (6.11) is clear: all the diverging polar parts of $\mathscr{Z}(\mathscr{G})$ are removed by simply factorizing out the proper reduced partition functions (6.12) of the $L$-star walks, as many times $n_{L}$ as an $L$-vertex appears in $\mathscr{G}$. These polar parts scale with $S$ with a set of irreducible independent critical exponents. We have indeed [45] for $z \rightarrow \infty$ and $d<4$,

$$
\hat{Z}_{L}(z, d) \sim z^{\sigma_{L} /(2-d / 2)} \sim S^{\sigma_{L}}, \quad z \rightarrow \infty,
$$

where $\sigma_{L}$ is a irreducible universal critical exponent associated with the vertex of $L$ walks. (Note that for polymers, i.e. usual SAW's, the exact value of $\sigma_{L}$ has been obtained [45] in two dimensions $\sigma_{L}=(2-L)(9 L+2) / 64$. From this set of values, one can derive new infinities of exact geometrical exponents for SAW's [45, 48].)

Here we have for a star walk (Fig. 13), according to Eqs. (6.9), (6.10), (6.12)

$$
\hat{Z}_{L}(z, d) \equiv \mathscr{Z}\left(\mathscr{S}_{L}\right) \equiv Z_{\mathscr{S}_{L}}(z, d) \sim S^{\sigma_{L}}
$$


Hence $\sigma_{L}$ is also the scaling exponent of the $L$ star walk. Because of Eq. (6.10), we have immediately

$$
\sigma_{1} \equiv 0
$$

The overall scaling behaviour of $\mathscr{Z}(\mathscr{G})(6.11)$ is now obtained from $(6.13),(6.14)$

$$
\mathscr{Z}(\mathscr{G}) \sim S^{-d \mathscr{L} / 2+\sum_{L \geqq 1} n_{L} \sigma_{L}} A_{\mathscr{G}}^{*}, \quad S \rightarrow \infty
$$

We only need now to calculate the star partition functions $\mathscr{Z}\left(\mathscr{S}_{L}\right)$, which will yield the basic exponents $\sigma_{L}$ 's.

\section{6.c. Logarithmic Behaviour in $d=4$}

For calculating the dominant logarithmic behaviours in $d=4$, a first order calculation is sufficient. In the next section the second order one will be performed to get $O\left(\varepsilon^{2}\right)$ results. The combinatorial calculation of $\mathscr{Z}\left(\mathscr{S}_{L}\right)$ for a star walk is extremely easy to first order in $z$ [45] (Fig. 13) [Eq. (2.15)]

$$
\mathscr{Z}\left(\mathscr{S}_{L}\right)=1-\frac{2}{\varepsilon} z \frac{1}{2} L(L-1)+\ldots
$$

The critical exponent $\sigma_{L}$ is then calculated as

$$
\sigma_{L}=S \frac{\partial}{\partial S} \ln \mathscr{Z}\left(\mathscr{P}_{L}\right)
$$

and reads to first order in $g$

$$
\sigma_{L}[g, \varepsilon]=-g \frac{1}{2} L(L-1)+O\left(g^{2}\right) .
$$

Of course it is regular to all orders in $g$ when $\varepsilon \rightarrow 0$. For $d=4-\varepsilon$, its fixed point value is thus from (2.22),

$$
\sigma_{L}=-\frac{\varepsilon}{8} L(L-1)+O\left(\varepsilon^{2}\right)
$$

Hence Eq. (6.17) implies that the probability $\mathscr{Z}(\mathscr{G})$ of no further intersections of the net of walks $\mathscr{G}$ scales like

with

$$
\mathscr{Z}(\mathscr{G}) \sim(2 \pi S)^{-\mathscr{L} d / 2} S^{\zeta_{\mathscr{G}}} \quad(S \rightarrow \infty)
$$

$$
\zeta_{\mathscr{G}}=-\sum_{L \geqq 1} n_{L} \frac{\varepsilon}{8} L(L-1)+O\left(\varepsilon^{2}\right)
$$

In $d=4$, we write instead (6.19) as

$$
\frac{\partial}{\partial g} \ln \mathscr{Z}\left(\mathscr{S}_{L}\right)=\sigma_{L}[g, 0] / W[g, 0]
$$


and get from (2.21) and (6.20),

Hence

$$
\frac{\partial}{\partial g} \ln \mathscr{Z}\left(\mathscr{S}_{L}\right)=\frac{1}{4 g} L(L-1)+O(1) .
$$

$$
\hat{Z}_{L} \equiv \mathscr{Z}\left(\mathscr{S}_{L}\right)=C_{L} g^{\frac{1}{4} L(L-1)}[1+O(g)]
$$

where $C_{L}$ is a non-universal constant. Now we insert this information inside (6.11), together with (6.13bis), to get in $4 D$,

$$
\mathscr{Z}(\mathscr{G}) \sim(2 \pi S)^{-2 \mathscr{L}} g_{L \geqq 1} n_{L} L(L-1) / 4[1+O(g)] .
$$

We can use the refined result (5.17bis) for $g$ in $d=4$, to get the exact asymptotic behaviour in $\mathbb{Z}^{4}$

$$
\begin{aligned}
\mathscr{Z}(\mathscr{G}) \sim(2 \pi S)^{-2 \mathscr{L}}\left(\frac{1}{2 \ln S}\right)^{\frac{1}{4}} \sum_{L \geqq 1} n_{L} L(L-1) \\
\cdot\left[1+\frac{\ln \ln S}{\ln S} \frac{1}{4} \sum_{L \geqq 1} n_{L} L(L-1)+O\left(\ln ^{-1} S\right)\right] .
\end{aligned}
$$

This yields the full generalization of Lawler's result to any net of walks. For the case (6.2) of two walks, we naturally recover (5.22). Equation (6.25) gives the answer in $\mathbb{Z}^{4}$ to question (6.1). We remark that the $L=1$ vertices do not contribute to the scaling behaviour. The origin of this fact lies in Eq. (6.10).

\section{6.d. Calculation to $O\left(\varepsilon^{2}\right)$}

For completeness, let us now calculate to order $O\left(\varepsilon^{2}\right)$ the set of basic critical exponents $\sigma_{L}$ governing the partition functions $\mathscr{Z}\left(\mathscr{S}_{L}\right)$. The diagrams contributing to this order are given in Fig. 14, together with their values. Note that the last diagram of Fig. 14 is the only one which has not been calculated above. It equals $\mathscr{I}_{2}$ (5.8). It remains to attribute to each diagram its combinatorial weight, obtained by choosing the interactions between the $L$ chains. We find finally

$$
\mathscr{Z}\left(\mathscr{S}_{L}\right)=1+\left(\begin{array}{l}
L \\
2
\end{array}\right) \mathscr{I}+\frac{1}{2}\left(\begin{array}{c}
L \\
2
\end{array}\right)\left(\begin{array}{c}
L-2 \\
2
\end{array}\right) \mathscr{I}^{2}+\left(\begin{array}{l}
L \\
2
\end{array}\right) \mathscr{I}_{1}+\left[\left(\begin{array}{c}
L \\
2
\end{array}\right)+2 L\left(\begin{array}{c}
L-1 \\
2
\end{array}\right)\right] \mathscr{I}_{2} .
$$

Inserting the elementary values

$$
\begin{aligned}
& \left.\mathscr{I}=z\left(-\frac{2}{\varepsilon}-1+\ln 2\right)\right), \\
& \mathscr{I}_{1}=z^{2}\left[\frac{4}{\varepsilon^{2}}+\frac{4}{\varepsilon}(1-\ln 2)\right], \\
& \mathscr{I}_{2}=z^{2}\left[\frac{2}{\varepsilon^{2}}+\frac{1}{\varepsilon}(3-2 \ln 2)\right],
\end{aligned}
$$




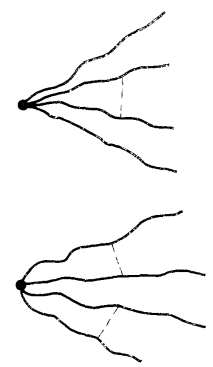

$$
y=z\left(-\frac{2}{\varepsilon}-1+\ln 2\right)
$$

b) 2

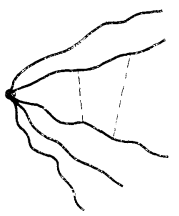

$3 y_{1}=z^{2}\left[\frac{4}{\varepsilon^{2}}+\frac{4}{\varepsilon}(1-\ln 2)\right]$

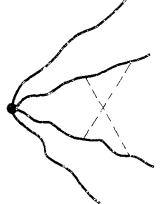

$$
y_{2}=z^{2}\left[\frac{2}{\varepsilon^{2}}+\frac{1}{\varepsilon}(3-2 \ln 2)\right]
$$

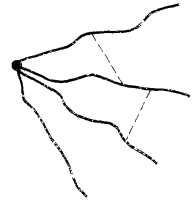

by,

Fig. 14. Diagrams contributing to $\mathscr{Z}\left(\mathscr{S}_{L}\right)$ for $L$ walks up to two loops, and their nomina1 contributions. Here $L=4$, but for $L<3$ some diagrams do not appear. This is taken into account by attributing to each diagram its combinatorial weight, which is $L$ dependent [see Eq. (6.26)]

we find

$$
\begin{aligned}
\mathscr{Z}\left(\mathscr{S}_{L}\right) & \equiv \hat{Z}_{L}(z, d)=1+z\left[-\frac{2}{\varepsilon}\left(\begin{array}{l}
L \\
2
\end{array}\right)+(\ln 2-1)\left(\begin{array}{l}
L \\
2
\end{array}\right)\right] \\
& +z^{2}\left\{\frac{1}{\varepsilon^{2}}\left[6\left(\begin{array}{c}
L \\
2
\end{array}\right)+4 L\left(\begin{array}{c}
L-1 \\
2
\end{array}\right)+2\left(\begin{array}{c}
L \\
2
\end{array}\right)\left(\begin{array}{c}
L-2 \\
2
\end{array}\right)\right]\right. \\
& \left.+\frac{1}{\varepsilon}\left[\left(\begin{array}{l}
L \\
2
\end{array}\right)(7-6 \ln 2)+2 L\left(\begin{array}{c}
L-1 \\
2
\end{array}\right)(3-2 \ln 2)+2\left(\begin{array}{c}
L \\
2
\end{array}\right)\left(\begin{array}{c}
L-2 \\
2
\end{array}\right)(1-\ln 2)\right]\right\} .
\end{aligned}
$$

Let us now simplify the notations

$$
\hat{Z}_{L}=\mathscr{Z}\left(\mathscr{S}_{L}\right)=1+z\left(\frac{a}{\varepsilon}+a^{\prime}\right)+z^{2}\left(\frac{b}{\varepsilon^{2}}+\frac{b^{\prime}}{\varepsilon}\right),
$$


where $a, a^{\prime}, b, b^{\prime}$ can be read off from $(6.27 a)$ :

$$
\begin{aligned}
& a=-2\left(\begin{array}{l}
L \\
2
\end{array}\right), \quad a^{\prime}=(\ln 2-1)\left(\begin{array}{l}
L \\
2
\end{array}\right), \\
& b=6\left(\begin{array}{l}
L \\
2
\end{array}\right)+4 L\left(\begin{array}{c}
L-1 \\
2
\end{array}\right)+2\left(\begin{array}{l}
L \\
2
\end{array}\right)\left(\begin{array}{c}
L-2 \\
2
\end{array}\right), \\
& b^{\prime}=\left(\begin{array}{l}
L \\
2
\end{array}\right)(7-6 \ln 2)+2 L\left(\begin{array}{c}
L-1 \\
2
\end{array}\right)(3-2 \ln 2)+2\left(\begin{array}{l}
L \\
2
\end{array}\right)\left(\begin{array}{c}
L-2 \\
2
\end{array}\right)(1-\ln 2) .
\end{aligned}
$$

For calculating $\sigma_{L}(6.19)$ we can substitute $g(5.11)$ to $z$ in

$$
\sigma_{L}(z, \varepsilon)=\frac{\varepsilon}{2} z \frac{\partial}{\partial z} \ln \hat{Z}_{L}
$$

and obtain a regular scaling function $\sigma_{L}[g, \varepsilon]$. We prefer to use here a different method, which is simpler, and on which we would like to draw the attention of the reader. In [34], we have indeed shown (in the case of real polymers) that rather than substituting the second virial coefficient $g$ to $z$ as in [18], one can use equivalently a simpler minimal subtraction scheme. We showed that it exists a unique renormalized variable $z_{R}$ with the minimal double Laurent-Taylor expansion of $z\left(z_{R}, c\right)$,

$$
z=\sum_{n \geqq 1} z_{R}^{n} \sum_{y=0}^{n-1} \frac{a_{n, p}}{\varepsilon^{n-p}}
$$

where the coefficients $a_{n, p}$ are pure numbers independent of $\varepsilon$ and $z$, such that any scaling function like $g(z, \varepsilon), \zeta(z, \varepsilon), \sigma_{L}(z, \varepsilon)$ above, etc. .., becomes regular with respect to $\varepsilon, \varepsilon \rightarrow 0$, to ail orders in $z_{R}$. For the present case of intersections of random walks, a similar minimal scheme (6.29) exists. It has the advantage over $g(5.11)$, to be free of irrelevant finite contributions, $g$ and $z_{R}$ are related together by a finite renormalization $[20,34]$. Let us impose for instance that $g(5.11)$ for two random walks,

$$
g=z+z^{2}\left(-\frac{4}{\varepsilon}-1+4 \ln 2\right)+z^{3}\left[\frac{16}{\varepsilon^{2}}+\frac{1}{\varepsilon}(12-32 \ln 2)\right],
$$

is regular in $\left(z_{R}, \varepsilon\right)$. We find immediately the universal expansion (6.29) of $z$

$$
z=z_{R}+\frac{4}{\varepsilon} z_{R}^{2}+\left(\frac{16}{\varepsilon^{2}}-\frac{4}{\varepsilon}\right) z_{R}^{3}+\ldots .
$$

Note that this minimal expansion is simpler than the equivalent one (5.12) in terms of $g$ and makes calculations easier. The fixed point value of $z_{R}$ is obtained $[20,34]$ by defining a Wilson function

$$
W(z, \varepsilon)=W\left[z_{R}, \varepsilon\right]=\frac{\check{\varepsilon}}{2} z \frac{\partial}{\partial z} z_{R},
$$

and solving $W\left[z_{R}^{*}, \varepsilon\right]=0$. We find

$$
z_{R}^{*}=\frac{\varepsilon}{4}+2\left(\frac{\varepsilon}{4}\right)^{2}+O\left(\varepsilon^{3}\right)
$$


This gives the universal "minimal" fixed point for intersections of two random walks in $\mathbb{R}^{d}, d<4$, and can be used in any further study.

Let us now calculate $\sigma_{L}(6.28),(6.27 \mathrm{~b})$ with this scheme. Substituting $z_{R}$ to $z$ with (6.30) gives

$$
\sigma_{L}\left[z_{R}, \varepsilon\right]=\left(\frac{a}{2}+a^{\prime} \frac{\varepsilon}{2}\right) z_{R}+z_{R}^{2}\left[\left(2 a+b-\frac{a^{2}}{2}\right) \frac{1}{\varepsilon}+2 a^{\prime}+b^{\prime}-a a^{\prime}\right] .
$$

The renormalizability of the theory requires first the following identity to hold true:

$$
2 a+b-\frac{a^{2}}{2}=0
$$

It can be indeed checked from (6.27c), as an identity valid for any $L \geqq 1$. Now, we find the fixed point value $\sigma_{L}\left[z_{R}^{*}, \varepsilon\right]$ from $(6.31)$,

$$
\sigma_{L}\left[z_{R}^{*}, \varepsilon\right]=\frac{a}{2} \frac{\varepsilon}{4}+\left(a+4 a^{\prime}+b^{\prime}-a a^{\prime}\right)\left(\frac{\varepsilon}{4}\right)^{2}+O\left(\varepsilon^{3}\right) .
$$

The particular values $(6.27 \mathrm{c})$ of $a, a^{\prime}, b, b^{\prime}$ yield, after some simplifications, the universal exponent

$$
\sigma_{L}=-\frac{1}{2} L(L-1) \frac{\varepsilon}{4}+\frac{1}{2} L(L-1)(2 L-5)\left(\frac{\varepsilon}{4}\right)^{2}+O\left(\varepsilon^{3}\right)
$$

which is our final result.

One notices that all the $\ln 2$ terms appearing in the expansion (6.27a) have disappeared in this critical exponent. Mathematically, this comes from the fact that the coefficient found for $\ln 2$ in $\sigma_{L}$ is precisely (6.33), which must vanish for the renormalization to be valid. Physically, the occurrence of $\ln 2$ terms is an artefact of the direct renormalization method, where finite walks of fixed lengths are considered. This induces boundary effects (i.e. $\ln 2$ terms), which are absent in the field theoretic version of the same critical theory. In the latter case, all the lengths of the walks fluctuate and only the total length is controlled exponentially by a mass term or a "killing time". We note that universality requires the critical exponents to be the same. Here, this is insured by (6.33), which is a renormalizability condition. Hence renormalizability and universality are synonyms. This appearance of boundary effect is the drawback of direct renormalization [18] and makes calculations slightly more complicated (see comment [43]). These effects disappear in critical exponents. However, they are of importance in some universal numbers like $g$ (5.14).

The value $(6.35)$ gives the critical exponent $\zeta_{\mathscr{G}}$ governing the probability $\mathscr{Z}(\mathscr{G})$ for any $\mathscr{G}$

$$
\mathscr{Z}(\mathscr{G}) \sim S^{-\frac{d}{2} \mathscr{L}+\zeta_{\mathscr{G}}}, \quad S \rightarrow \infty
$$

with

$$
\zeta_{\mathscr{G}}=\sum_{L \geqq 1} n_{L} \sigma_{L}=\sum_{L \geqq 1} n_{L} \frac{1}{2} L(L-1)\left\{-\frac{\varepsilon}{4}+(2 L-5)\left(\frac{\varepsilon}{4}\right)^{2}+O\left(\varepsilon^{3}\right)\right\}
$$


We remark again that the $L=1$ vertices, i.e. the origins or ends of walks do not contribute to $\zeta_{\mathscr{G}}$, and this of course is valid to all orders in $\varepsilon$, since it comes directly from (6.10).

\section{6.e. Three-Walk Crossings}

We have considered above the exclusion of further two-walk intersections between any two random walks in the net of walks $\mathscr{G}$. One can of course consider the problem of excluding three-walk intersections only, the two-walk ones being allowed. This obviously generalizes the case considered in Sect. 3 for three walks starting at the origin. The critical dimension for this tricritical problem [40] is still of course $d=3$. Using the formalism developed in Sect. 3, and extending the analysis given in Sects. $6 a-c$ above, to the tricritical case, it is clear that we can evaluate $\mathscr{Z}(\mathscr{G})$. We shall not give further details of calculations here. The general results read:

$$
\text { For } \quad d=3-\varepsilon^{\prime}, \varepsilon^{\prime}>0, \quad \mathscr{Z}(\mathscr{G}) \sim(2 \pi S)^{-\mathscr{L} d / 2} S^{\zeta_{\mathscr{G}}},
$$

where $\zeta_{\mathscr{G}}$ is the universal exponent

$$
\zeta_{\mathscr{G}}=-\frac{\varepsilon^{\prime}}{24} \sum_{L \geqq 3} n_{L} L(L-1)(L-2)+O\left(\varepsilon^{\prime 2}\right) .
$$

For $d=3$, the universal limit is

$$
\mathscr{Z}(\mathscr{G}) \approx(2 \pi S)^{-\frac{3}{2} \mathscr{L}}(\ln S)^{-\frac{1}{24}} \sum_{L \geqq 3} n_{L} L(L-1)(L-2) .
$$

For the simple Lawler's case of three walks starting at the same point: $n_{1}=3, n_{3}=1$, and we do recover (3.25), as expected.

We can also generalize these results to a net $\mathscr{G}$ in $d$ dimensions, for $d \leqq d^{*}$ $=2 P /(P-1)$, when only further $P$-walk simultaneous intersections are forbidden. In this case we have for any number $L$ of walks $1 \leqq L \leqq P-1$,

$$
\mathscr{Z}\left(\mathscr{S}_{L}\right) \equiv 1,
$$

since there are no possible $P$-walk intersections for $L<P$. Furthermore, we have for $L=P$ walks

$$
\mathscr{Z}\left(\mathscr{S}_{P}\right) \equiv \mathscr{Z},
$$

where $\mathscr{Z}(4.5)$ is the probability of no intersection of $P$ walks starting at the origin calculated in Sect. 4. Hence in all generality, we have

$$
\zeta_{P} \equiv \sigma_{P}
$$

where $\zeta_{P}$ is defined in (4.24) and $\sigma_{P}$ in (6.16). Therefore, from (4.26) we find to first order in $\varepsilon=\frac{2 P}{P-1}-d$ the fixed point value:

$$
\sigma_{P}=-\frac{\varepsilon}{2^{P}}(P-1)+O\left(\varepsilon^{2}\right)
$$


and the scaling function $(4.25)$ in $d=d^{*}=\frac{2 P}{p-1}$,

$$
\sigma_{P}[g, 0]=-(P-1) \Gamma^{P-1}\left(\frac{1}{P-1}\right) g+O\left(g^{2}\right)
$$

Now, to first order in $g$ or $\varepsilon$, it is easy to calculate $\sigma_{L}$ for $L \geqq P$ in terms of the scaling function for $P$ walks. Looking indeed at the first order diagram of $L$ walks $(L \geqq P$ ) with one intersection of $P$ walks, one gets a new combinatorial weight $\left(\begin{array}{l}L \\ P\end{array}\right)=\frac{L(L-1) \ldots(L-P+1)}{P !}$, which is the number of choices of $P$ walks among $L$. Therefore, comparing to Eq. (4.15), we find immediately

$$
\mathscr{Z}\left(\mathscr{S}_{L}\right)=1-z\left(\begin{array}{l}
L \\
P
\end{array}\right) \frac{2}{\varepsilon} \Gamma^{P-1}\left(\frac{1}{P-1}\right)+O\left(z^{2}\right)
$$

From this, we deduce the relation between scaling functions (4.24) and (6.19) in the case of $P$-body intersections, valid only to first order,

$$
\sigma_{L}[g, \varepsilon]=\left(\begin{array}{l}
L \\
P
\end{array}\right) \sigma_{P}[g, \varepsilon]+O\left(g^{2}\right)
$$

Owing to (6.42) this gives the fixed point value

$$
\sigma_{L}=-\frac{L(L-1) \ldots(L-P+1)}{P !}(P-1) \frac{\varepsilon}{2^{P}}+O\left(\varepsilon^{2}\right)
$$

and from (6.43) in $d^{*}$ dimensions, the scaling function

$$
\sigma_{L}[g, 0]=-\frac{L(L-1) \ldots(L-P+1)}{P !}(P-1) \Gamma^{P-1}\left(\frac{1}{P-1}\right) g+O\left(g^{2}\right) .
$$

Now, from the general multiplicative equation (6.11), (6.16), (6.17), the scaling behaviour of the probability $\mathscr{Z}(\mathscr{G})$ is found for any net of walks $\mathscr{G}$ in $d \leqq d^{*}$ dimensions, with $P$-point intersection forbidden. First for $d<d^{*}$, it reads

with

$$
\mathscr{Z}(\mathscr{G}) \sim S^{-\mathscr{L} d / 2+\zeta_{\mathscr{G}}}
$$

$$
\zeta_{\mathscr{G}}=\sum_{L \geqq P} n_{L} \sigma_{L}=-\sum_{L \geqq P} n_{L} \frac{L(L-1) \ldots(L-P+1)}{P !} \frac{P-1}{2^{P}} \varepsilon+O\left(\varepsilon^{2}\right)
$$

At the critical dimension $d=d^{*}$, one has to calculate the $g$-derivative

$$
\frac{\partial}{\partial g} \ln \mathscr{Z}\left(\mathscr{P}_{L}\right)=\sigma_{L}[g, 0] / W[g, 0]=\left(\begin{array}{l}
L \\
P
\end{array}\right) \frac{1}{g} \frac{1}{2^{P-1}}+O(1)
$$

where use has been made of (6.45) and (4.19bis), (4.21 bis).

Integration gives

$$
\mathscr{Z}\left(\mathscr{S}_{L}\right) \approx g\left(\begin{array}{l}
L \\
P
\end{array}\right) \frac{1}{2^{P-1}}
$$


Inserting this into (6.11), we find finally

$$
\mathscr{Z}(\mathscr{G}) \approx S^{-\frac{d^{*}}{2} \mathscr{L}}(\ln S)^{3_{\mathscr{G}}}
$$

with the exact critical logarithmic exponents

$$
3_{\mathscr{G}}=-\sum_{L \geqq P} n_{L}\left(\begin{array}{l}
L \\
P
\end{array}\right) \frac{1}{2^{P-1}} .
$$

This gives the exact logarithmic scaling behaviour for any net of walks in $d^{*}=\frac{2 P}{P-1}$ dimensions when $P$-walk mutual intersections are forbidden. For $P=2,3$ one recovers from Eqs. (6.46) and (6.48), the former results (6.22) (6.25) for $P=2$, and (6.37), (6.38) for $P=3$. QED

In conclusion. we have shown in this article how various intersection probabilities for random walks in $\mathbb{Z}^{d}$ or Brownian paths in $\mathbb{R}^{d}$ can be calculated from a minimal continuum model of the Edwards' type. This included the study of double points in $d \leqq 4$, triple points in $d \leqq 3, \ldots, P$-tuple points in $d \leqq 2 P /(P-1)$. The essential analytical tools are: perturbation expansion around the Brownian paths in series of the number of intersections, study of the singular parts of the perturbation series, followed by a direct renormalization, which actually amounts to resum the dominant scaling behaviour of the probabilities or partition functions. The method is quite flexible and can be applied to any topological problem of intersections. In particular, we generalized considerably Lawler's results to the study of any net of walks which form a hydrographical network with a prescribed set of confluence points. The exact irreducible universal contributions of the star walks, i.e. the confluence points, were given at order $O\left(\varepsilon^{2}\right)$, as well as the corresponding irreducible logarithmic factors in $d=4$.

The perturbation technique around Brownian paths is originally close from the one used in probabilistic rigorous studies. However, the (direct) multiplicative renormalization scheme is not considered or known there. So we hope that this study could help to make this now standard methods of theoretical physics more directly available to mathematicians.

Acknowledgement. We thank G.F. Lawler for an interesting correspondence after he received this work.

\section{References}

1. Dvoretzky, A., Erdös, P., Kakutani, S.: Double points of Brownian paths in n-space. Acta Sci. Math. 12, 75-81 (1950)

2. Erdös, P., Taylor, S.J. : Some problems concerning the structure of random walk paths. Acta Math. Acad. Sci. Hung. 11, 137-162 (1960)

3. Erdös, P., Taylor, S.J.: Some intersection properties of random walk paths. Acta Math. Acad. Sci. Hung. 11, 231-248 (1960) 
4. Lawler, G.F.: A self-avoiding random walk. Duke Math. J. 47, 655-693 (1980)

5. Brydges, D., Spencer, T.: Self-avoiding walks in 5 or more dimensions. Commun. Math. Phys. 97, 125 (1985)

6. Aizenman, M.: Geometric analysis of $\phi^{4}$ fields and Ising models. Parts I and II. Commun. Math. Phys. 86, 1 (1982)

7. Brydges, D.C., Fröhlich, J., Spencer, T.: The random walk representation of classical spin systems and correlation inequalities. Commun. Math. Phys. 83, 123 (1982)

Brydges, D.C., Fröhlich, J., Sokal, A.D.: The random walk representation of classical spin systems and correlation inequalities. II. The Skeleton inequalities. Commun. Math. Phys. 91, 117 (1983)

Brydges, D.C., Fröhlich, J., Sokal, A.D. : A new proof of the existence and nontriviality of the continuum $\varphi_{2}^{4}$ and $\varphi_{3}^{4}$ quantum field theories. Commun. Math. Phys. 91, 141 (1983)

8. Aragão de Carvalho, C., Caracciolo, S., Fröhlich, J.: Polymers and $g|\phi|^{4}$ theory in four dimensions. Nucl. Phys. B215 [FS7], 209 (1983)

9. Aizenman, M.: The intersection of Brownian paths as a case study of a renormalization group method for quantum field theory. Commun. Math. Phys. 97, 91 (1985)

10. Felder, G., Fröhlich, J.: Intersection properties of simple random walks : a renormalization group approach. Commun. Math. Phys. 97, 111 (1985)

11. Lawler, G.F.: The probability of intersection of independent random walks in four dimensions. Commun. Math. Phys. 86, 539-554 (1982)

12. Lawler, G.F.: Intersections of random walks in four dimensions. II. Commun. Math. Phys. 97, 583-594 (1985); Intersections of simple random walks. Contemp. Math. 41, 281-289 (1985)

13. Lawler, G.F.: The probability of intersection of three random walks in three dimensions. Duke University Preprint

14. Le Gall, J.-F.: Propriétés d'intersection des marches aléatoires. I. Convergence vers le temps local d'intersection. Commun. Math. Phys. 104, 471 (1986)

15. Le Gall, J.-F.: Propriétés d'intersection des marches aléatoires. II. Etude des cas critiques. Commun. Math. Phys. 104, 505-524 (1986)

16. Rosen, J.: Random walks and intersection local time (to be published)

17. Yor, M.: Renormalisation et convergence en loi pour les temps locaux d'intersection du mouvement brownien dans $\mathbb{R}^{3}$. In: Séminaire de probabilités XIX. Lecture Notes in Mathematics, Vol. 1123, pp. 350-365. Berlin, Heidelberg, New York: Springer 1985, and references theirein

18. des Cloizeaux, J.: Polymers in solution: Principles and applications of a direct renormalization method. J. Phys. 42, 635 (1981)

19. Duplantier, B. : Critical amplitudes of Edwards' continuous polymer chain model. J. Phys. 47, 1865 (1986)

20. Duplantier, B.: Polymer chains in four dimensions. Nucl. Phys. B 275 [FS17], 319 (1986)

21. de Gennes, P.G.: Scaling concepts in polymer physics. Ithaca, London, Cornell University Press 1979

22. Joanny, J.F., Leibler, L., Ball, R. : Is chemical mismatch important in polymer solutions? J. Chem. Phys. 81, 4640 (1984)

23. de Gennes, P.G.: Exponents for the excluded volume problem as derived by the Wilson method. Phys. Lett. 38 A, 339 (1972)

24. des Cloizeaux, J.: The Lagrangian theory of polymer solutions at intermediate concentration. J. Physique 36, 281 (1975)

25. Duplantier, B.: Chaînes polymères et champ à zéro composante. C.R. Acad. Sci. Paris 290 B, 199 (1980)

26. Symanzik, K.: Euclidean quantum field theory. In: Local quantum theory. Jost R. (ed.). New York, London: Academic Press 1969

27. Phase Transitions and Critical Phenomena. Domb, C., Green, M.S. (eds), Vol. 6, New York, London: Academic Press 1976

28. Edwards, S.F.: The statistical mechanics of polymers with excluded volume. Proc. Phys. Soc. 85, $613(1965)$ 
29. Westwater, J. : On Edwards' model for polymer chains. Commun. Math. Phys. 72, 131-174 (1980)

30. Westwater, J. : On Edwards' model for polymer chains. II. The self-consistent case. Commun. Math. Phys. 79, 53-73 (1981)

31. Westwater, J.: On Edwards' model for polymer chains. III. Borel summability. Commun. Math. Phys. 84, 459-470 (1982)

32. Westwater, J.: On Edwards' model for polymer chains. In: Bielefeld Encounters in Mathematics and Physics IV. Albeverio, S., Blanchard, Ph. (eds.). Singapore: World Scientific Publishing 1984

33. Benhamou, M., Mahoux, G.: Multiplicative renormalization of continuous polymer theories, in good and $\theta$ solvents, up to critical dimensions. J. Phys. 47, 559 (1986)

34. Duplantier, B.: Dimensional renormalizations of polymer theory. J. Phys. 47, 569 (1986)

35. Duplantier, B. Integral representation of the dimensionally renormalized polymer partition functions. Europhys. Lett. 1, 99 (1986)

36. Wolpert, R.: Wiener path intersections and local time. J. Funct. Anal. 30, 329 (1978)

37. Geman, D., Horowitz, J., Rosen, J. : A local time analysis of intersections of Brownian paths in the plane. Ann. Probab. 12, 86 (1984)

38. For a discussion in field theory see, e.g., Bergère, M., David, F.: Integral representation for the dimensionally regularized massive Feynman amplitude. J. Math. Phys. 20, 1244 (1979)

39. Duplantier, B. : Lagrangian tricritical theory of polymer chain solutions near the $\theta$-point. J. Phys. 43, 991 (1982)

40. Duplantier, B.: Tricritical polymer chains in or below three dimensions, Europhys. Lett. 1, 491 (1986)

41. Duplantier, B.: Direct or dimensional renormalizations of the tricritical polymer theory. J. Phys. 47, 745 (1986)

42. Duplantier, B.: Geometry of polymer chains near the theta-point and dimensional regularization. J. Chem. Phys. 86, 4233 (1987)

43. The best procedure is to represent each $\theta$ function in (5.4) (5.5) as an imaginary contour integral $\theta(x)=\int_{\mathscr{E}} e^{a x} \frac{d a}{2 \pi i a}$, where $\mathscr{C}$ lies on the right-hand side of the origin in $\mathbb{C}$. This reconstructs a Schwinger-Feynmann $\alpha$-parameter representation of the field theory, where now two different masses occur. Then one can use standard treatments of such integrals, by adapting them to the more complicated case of multiple masses. For a treatment in the symmetric case, see Zinn-Justin, J. (1987, in preparation)

44. Cates, M.E., Witten, T.A.: Diffusion near absorbing fractals: Harmonic measure exponents for polymers. Phys. Rev. A 35, 1809 (1987)

45. Duplantier, B.: Polymer network of fixed topology: Renormalization, exact critical exponent $\gamma$ in two dimensions and $d=4-\varepsilon$. Phys. Rev. Lett. 57, 941 (1986)

46. Fisher, M.E.: Walks, walls, wetting and melting. J. Stat. Phys. 34, 667 (1984)

47. Huse, D.A., Fisher, M.E.: Commensurate melting, domain walls, and dislocations. Phys. Rev. B29, 239 (1984)

48. Duplantier, B.: Exact contact critical exponents of a self-avoiding polymer chain in two dimensions. Phys. Rev. B35, 5290 (1987)

Communicated by J. Fröhlich

Received November 8, 1987 
